\title{
Gemini IFU, VLA, and HST observations of the OH Megamaser Galaxy IRAS17526 + 3253*
}

\author{
Dinalva A. Sales ${ }^{\oplus},{ }^{1} \dagger$ A. Robinson, ${ }^{2,3} \dagger$ R. A. Riffel ${ }^{\oplus},{ }^{4} \dagger$ T. Storchi-Bergmann, ${ }^{5}$ J. \\ F. Gallimore, ${ }^{6}$ P. Kharb ${ }^{\oplus}{ }^{7}$ S. Baum, ${ }^{3,8}$ C. O’ Dea ${ }^{\oplus},{ }^{2,8}$ C. Hekatelyne ${ }^{4}$ and F. Ferrari ${ }^{\oplus 1}$ \\ ${ }^{1}$ Universidade Federal do Rio Grande, Instituto de Matemática, Estatística e Física, 8 km Itália Av., 96203-900 Rio Grande, RS, Brazil \\ ${ }^{2}$ School of Physics and Astronomy, Rochester Institute of Technology, 84 Lomb Memorial Drive, Rochester, NY 14623, USA \\ ${ }^{3}$ Chester F. Carlson Center for Imaging Science, Rochester Institute of Technology, 54 Lomb Memorial Drive, Rochester, NY 14623, USA \\ ${ }^{4}$ Universidade Federal de Santa Maria, Departamento de Física, Centro de Ciências Naturais e Exatas, 97105-900 Santa Maria, RS, Brazil \\ ${ }^{5}$ Departamento de Astronomia, Universidade Federal do Rio Grande do Sul. 9500 Bento Gonçalves, Porto Alegre 91501-970, Brazil \\ ${ }^{6}$ Department of Physics and Astronomy, Bucknell University, Lewisburg, PA 17837, USA \\ ${ }^{7}$ National Centre for Radio Astrophysics, Tata Institute of Fundamental Research, Pune University Campus, Ganeshkhind, 411007 Pune, India \\ ${ }^{8}$ Department of Physics and Astronomy, University of Manitoba, Winnipeg, MB R3T 2N2, Canada
}

Accepted 2019 January 4. Received 2018 December 17; in original form 2018 July 25

\begin{abstract}
We present a multiwavelength study of the $\mathrm{OH}$ megamaser galaxy IRAS17526 + 3253, based on new Gemini multi-object spectrograph integral field unit (GMOS/IFU) observations, Hubble Space Telescope F814W, and $\mathrm{H} \alpha+[\mathrm{N} \mathrm{II}]$ images, and archival 2MASS and $1.49 \mathrm{GHz}$ VLA data. The Hubble Space Telescope (HST) images clearly reveal a mid-to-advanced stage major merger whose northwestern and southeastern nuclei have a projected separation of $\sim 8.5 \mathrm{kpc}$. Our $H S T / \mathrm{H} \alpha+[\mathrm{N} \mathrm{II}]$ image shows regions of ongoing star formation across the envelope on $\sim 10 \mathrm{kpc}$ scales, which are aligned with radio features, supporting the interpretation that the radio emission originates from star-forming regions. The measured $\mathrm{H} \alpha$ luminosities imply that the unobscured star formation rate (SFR) is $\sim 10-30 \mathrm{M}_{\odot} \mathrm{yr}^{-1}$. The GMOS/IFU data reveal two structures in northwestern separated by $850 \mathrm{pc}$ and by a discontinuity in the velocity field of $\sim 200 \mathrm{~km} \mathrm{~s}^{-1}$. We associate the blueshifted and redshifted components with, respectively, the distorted disc of northwestern and tidal debris, possibly a tail originating in southeastern. Star formation is the main ionization source in both components, which have SFRs of $\sim 2.6-7.9 \mathrm{M}_{\odot} \mathrm{yr}^{-1}$ and $\sim 1.5-4.5 \mathrm{M}_{\odot} \mathrm{yr}^{-1}$, respectively. Fainter line emission bordering these main components is consistent with shock ionization at a velocity $\sim 200 \mathrm{~km} \mathrm{~s}^{-1}$ and may be the result of an interaction between the tidal tail and the northwestern galaxy's disc. IRAS17526 + 3253 is one of only a few systems known to host both luminous $\mathrm{OH}$ and $\mathrm{H}_{2} \mathrm{O}$ masers. The velocities of the $\mathrm{OH}$ and $\mathrm{H}_{2} \mathrm{O}$ maser lines suggest that they are associated with the northwestern and southeastern galaxies, respectively (Martin et al.; Wagner).
\end{abstract}

Key words: techniques: spectroscopic-galaxies: active-galaxies: evolution-galaxies: individual: IRAS17526 + 3253 - galaxies: interactions.

\section{INTRODUCTION}

Galaxy mergers are generally believed to play an important role in galaxy evolution, with the assembly of massive haloes via multiple

\footnotetext{
* This work is based partly on observations made with the NASA/ESA Hubble Space Telescope, obtained at the Space Telescope Science Institute, which is operated by the Association of Universities for Research in Astronomy, Inc., under NASA contract NAS 5-26555.

†E-mail: dinalvaires@gmail.com (DAS); axrsps@rit.edu (AR); rogemar@ufsm.br(RAR)
}

mergers being the central mechanism for structure formation in $\Lambda \mathrm{CDM}$ cosmologies (e.g. Blumenthal et al. 1984; Springel, Di Matteo \& Hernquist 2005; Boylan-Kolchin et al. 2009; Angulo et al. 2012). Observational evidence and cosmology simulations suggest that the most massive galaxies are formed in major mergers, whilst the growth of intermediate-mass galaxies is largely driven by minor mergers or cold gas accretion (e.g. reviews by Silk \& Mamon 2012; Kormendy \& Ho 2013; Conselice 2014). In this context, the population of optically faint but infrared luminous galaxies discovered by the Infrared Astronomical Satellite (IRAS) in the 1980's (Soifer et al. 1984) is of great interest since it includes many 
examples of gas-rich major mergers, or strong interactions (e.g. Sanders et al. 1988; Veilleux, Kim \& Sanders 2002; Haan et al. 2011). The immense infrared luminosities of these objects arise from dust heated by embedded starbursts and/or active galactic nuclei (AGN), both of which are thought to be triggered by gas inflows driven by tidal torques generated by the interaction (e.g. Barnes \& Hernquist 1992; Mihos \& Hernquist 1996; Hopkins et al. 2006). Two main subdivisions of this population are recognized: Luminous infrared galaxies (LIRGs) have far-infrared (FIR) luminosities of $L_{\text {FIR }} \gtrsim 10^{11} \mathrm{~L}_{\odot}$, whereas the most luminous systems, ultra-LIRGs (ULIRGs), have quasar-like luminosities of $L_{\mathrm{FIR}} \gtrsim 10^{12} \mathrm{~L}_{\odot}$ (see Sanders \& Mirabel 1996, for a review). It has been proposed that (U)LIRGs represent different phases in the evolution of gasrich mergers, with LIRGs evolving into ULIRGs as the merger progresses, eventually followed by the emergence of a luminous AGN in a massive elliptical host, as the remaining circum-nuclear gas and dust is dispersed by starburst and AGN-induced outflows (Sanders et al. 1988; Veilleux et al. 2002).

Many studies of local (U)LIRGs broadly support this idea. For example, merger morphology correlates with FIR luminosity, with advanced mergers becoming more prevalent at higher luminosities (e.g. Sanders et al. 1988; Veilleux et al. 2002; Hwang et al. 2010; Haan et al. 2011; Carpineti et al. 2015), and the fraction hosting powerful AGN increases with both FIR luminosity and merger stage (e.g. Veilleux et al. 2002, 2009; Nardini et al. 2010). At higher redshifts $(z \sim 1-2)$ (U)LIRGs are much more common than in the local universe (e.g. Caputi et al. 2007), and similar morphological trends are seen, although with a wider range of merger states at high luminosity (Kartaltepe et al. 2012). Beyond $z>$ 2, the morphological properties of the most luminous submillimeter galaxies (SMGs) are also consistent with mergers and interacting systems (e.g. Tacconi et al. 2008; Engel et al. 2010; Hayward et al. 2013). Massive outflows in neutral, ionized, and molecular gas have been detected in most ULIRGs, both in the local universe (e.g. Heckman et al. 2000; Rupke, Veilleux \& Sanders 2002; Martin 2005; Feruglio et al. 2010; Sturm et al. 2011; Rupke \& Veilleux 2013; Spoon et al. 2013; Veilleux et al. 2013; Cicone et al. 2014) and at higher redshifts in both ULIRGs (e.g. George et al. 2014) and SMGs (e.g. Banerji et al. 2011). (U)LIRGs are therefore crucial to understanding the role of mergers in galaxy evolution and black hole growth.

Integral field spectroscopy technic is a powerful tool to study these complex systems, providing spatially resolved information on gas and stellar kinematics and a range of spectral diagnostics. Using such information (for example), gas flows can be mapped and contributions of various ionization mechanisms (AGN or stellar photoionization, or shocks) can be inferred (e.g. Monreal-Ibero, Arribas \& Colina 2006; Monreal-Ibero et al. 2010; Rich et al. 2012; Rich, Kewley \& Dopita 2015).

Here, we present a high spatial resolution analysis of the morphology, gaseous excitation, and kinematics of the brightest radio nucleus of the merger system IRAS17526 + 3253. This system, which has a redshift $z=0.025$ (de Vaucouleurs et al. 1991), was first identified as a possible interacting galaxy by Andreasian \& Alloin (1994). In the Digitized Sky Survey image, it appears as a warped, elongated structure oriented approximately southeastern (SE)-northwestern (NW), and has two bright knots separated by $\approx 20 \operatorname{arcsec}$.

It has an FIR luminosity, as calculated from the 60 to $100 \mu \mathrm{m}$ IRAS fluxes, of $L_{\mathrm{FIR}}=10^{11.19} \mathrm{~L}_{\odot}$ (taken from The Imperial IRASFSC Redshift Catalogue; IIFSCz; Wang \& Rowan-Robinson 2009), which places it just above the lower limit for LIRGs. An optical spectrum obtained using a 2 arcsec wide slit by Baan, Salzer \& LeWinter (1998) shows line ratios characteristic of a starburst spectrum.

Baan \& Klöckner (2006) observed IRAS17526 + 3253 with the VLA A-array in the $L$ and $\mathrm{C}$ bands (1.49 and $4.9 \mathrm{GHz}$ ), finding that the radio source consists of a linear chain of knots, with three main components, spanning $\sim 30 \operatorname{arcsec}(\sim 15 \mathrm{kpc})$ and extending along PA $119^{\circ}$, closely aligned with the optical PA. These authors used three indicators to distinguish between AGN and starburst activity as the origin of the radio emission: the brightness temperature at $4.85 \mathrm{GHz}$, the FIR-radio flux ratio at $4.85 \mathrm{GHz}$, and the $1.4-4.8 \mathrm{GHz}$ spectral index, $\alpha_{4.8 \mathrm{GHz}}^{1.4 \mathrm{GHz}}$. Based on these, all three main components were classified as starburst-powered, although in the case of the NW component the peak spectral index has a value $S \propto v^{-0.25 \pm 0.22}$ (Baan \& Klöckner 2006), which may indicate a typical of AGN or the flat spectrum is due to te free-free emission (Gioia, Gregorini \& Klein 1982; Condon et al. 1983; Condon \& Broderick 1991).

Garwood, Dickey \& Helou (1987) were the first to look for the 1665 and $1667 \mathrm{MHz} \mathrm{OH}$ maser lines in IRAS17526 + 3253 but reported a non-detection. A detection was subsequently reported by Martin et al. (1989), with an integrated luminosity $\log \left(L_{\mathrm{OH}} / \mathrm{L}_{\odot}\right)$ $=0.99$ and recession velocity $\approx 7500 \mathrm{~km} \mathrm{~s}^{-1}$ (see also Martin 1989), although McBride \& Heiles (2013) were unable to confirm the detection in more recent Arecibo Telescope observations due to strong radio frequency interference. Martin (1989) also observed the H I $21 \mathrm{~cm}$ emission line in IRAS17526 + 3253, at a velocity $\approx 7800 \mathrm{~km} \mathrm{~s}^{-1}$, close to the heliocentric systemic velocity (7798 $\pm 9 \mathrm{~km} \mathrm{~s}^{-1}$; de Vaucouleurs et al. 1991) but redshifted by $\approx 300 \mathrm{~km} \mathrm{~s}^{-1}$ with respect to the $\mathrm{OH}$ maser. Molecular line observations by (Baan et al. 2008) detected $\mathrm{CO}$ emission in the ${ }^{12} \mathrm{CO}(1-0)$ and ${ }^{12} \mathrm{CO}(2-1)$ transitions, revealing (in the latter) two velocity components, a broad peak at $\approx 7500 \mathrm{~km} \mathrm{~s}^{-1}$ and a narrower peak at $\approx 7800 \mathrm{~km} \mathrm{~s}^{-1}$. IRAS $17526+3253$ is also known to host a $22 \mathrm{GHz} \mathrm{H}_{2} \mathrm{O}$ kilo-maser source (Wagner 2013), which exhibits a cluster of three narrow $\left(\approx 10 \mathrm{~km} \mathrm{~s}^{-1}\right)$ features at $\approx 7800 \mathrm{~km} \mathrm{~s}^{-1}$, close to the systemic velocity. The $\mathrm{H}_{2} \mathrm{O}$ maser lines have an integrated luminosity $\sim 360 \mathrm{~L}_{\odot}$ and possibly arise from a region of shocked gas.

Our study is based on new Hubble Space Telescope (HST)/Advanced Camera for Surveys (ACS) images and integral field spectroscopy obtained with the Gemini multi-object spectrograph integral field unit (GMOS/IFU) at the Gemini North Telescope. The paper is organized as follows. In Section 2, we describe the data-reduction procedures and the measurements derived from the HST imaging and GMOS/IFU data. Section 3 describes our results on the overall morphology of the IRAS17526 + 3253 system, and the gas excitation and kinematics within a $5^{\prime \prime} .1 \times 3$. 4 field of view (FOV) covering the NW of the two optical nuclei. We discuss the implications of our results in Section 4. The main results and conclusions are summarized in Section 5. Throughout this paper, we adopt the Hubble constant as $\mathrm{H}_{0}=70.5 \mathrm{~km} \mathrm{~s}^{-1} \mathrm{Mpc}^{-1}, \Omega_{\Lambda}=0.73$, and $\Omega_{m}=0.27$ (Planck Collaboration XVI 2013; Lahav \& Liddle 2014), corresponding to a scale at the galaxy of $497 \mathrm{pc} \operatorname{arcsec}^{-1}$.

\section{OBSERVATION AND DATA REDUCTION}

\subsection{Hubble Space Telescope images}

We acquired HST images of IRAS17526 + 3253 with the ACS, as part of a snapshot program of a large sample of $\mathrm{OH}$ Megamaser Galaxies (OHMGs; Program ID 11604; PI: D.J. Axon). The ACS 
wide-field channel (WFC) with broad (F814W), narrow (FR656N), and medium (FR914M) band filters was used.

The F814W filter was required to map the continuum morphology of the host galaxy. The ramp filter images were selected to cover the $\mathrm{H} \alpha$ line in the narrow-band filter and the nearby continuum in the medium-band filter. The FR656N ramp filter includes the $\mathrm{H} \alpha$ and the $[\mathrm{N}$ II] $] \lambda 6548,83$ lines at the redshift of IRAS17526 + 3253. The total integration times were 600s in the broad-band (I) F814W filter, 200s in the medium-band FR914M filter, and 600s in the narrow-band $\mathrm{H} \alpha$ FR656N filter.

We used the pipeline image products for further processing with the $\mathrm{IRAF}^{1}$ package. We removed cosmic rays from individual images

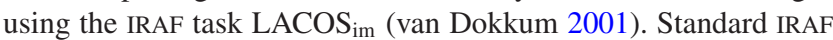
tasks were used to yield the final reduced images (see Fig. 1).

The continuum-free $\mathrm{H} \alpha+[\mathrm{N}$ II] image of IRAS17526 + 3253 was derived using the following procedure (see Hoopes, Walterbos \& Rand 1999; Rossa \& Dettmar 2000, 2003; Sales et al. 2015). First, we calculated the mean ratio of count rates, FR656N/FR914M, for several foreground stars that appear in both images. We then scaled the FR914M image by the mean count rate ratio and subtracted it from the FR656N image. The resulting, continuumsubtracted $\mathrm{H} \alpha+[\mathrm{N}$ II $]$ image was checked to verify that the residuals at the positions of the foreground stars were negligible compared to the expected noise level. This method gives typical uncertainties of 5-10 per cent (see Hoopes et al. 1999; Rossa \& Dettmar 2000, 2003).

\subsection{VLA radio data}

IRAS17526 + 3253 was observed at $1.49 \mathrm{GHz}$ using the VLA A-array configuration on 1990 March 7 (Project code: AM293). These data have previously been presented by Baan \& Klöckner (2006). We re-reduced these data following standard procedures in the Astronomical Imaging Processing System (AIPS). The final $1.49 \mathrm{GHz}$ VLA image with a beam-size of $1.35 \operatorname{arcsec} \times 1.11 \operatorname{arcsec}$ at a PA $=-39^{\circ}$ (Fig. 2) was obtained after several iterations of phase and amplitude self-calibration, using the AIPS tasks CALIB and IMAGR.

The final resultant rms noise in the image was $67 \mu \mathrm{Jy}_{\text {beam }}{ }^{-1}$. The radio image showing two unresolved cores, has been used to derive the astrometry of the HST, Gemini/IFU, and 2MASS Ks images

\subsection{Gemini multi-object spectrograph integral field unit data}

2D spectroscopic observations of IRAS17526 + 3253 were obtained using the GMOS/IFU (Allington-Smith et al. 2002). The observations were made at the Gemini North telescope on 2013 May, 15-16 UT, as part of program GS-2013A-Q-92. We used the IFU one-slit mode with the $\mathrm{B} 600+\mathrm{GG} 455$ grating, which encompassed the spectral range $\lambda \lambda 4855-7730$ and spectral resolution of $3.9 \AA$, covering an FOV of roughly $5^{\prime \prime} .1 \times 33^{\prime \prime} 4$. The IFU/FOV was centred on the complex of bright $\mathrm{H} \alpha+[\mathrm{N}$ II] emission associated with the northwestern (NW) galaxy of the interacting pair (Fig. 1) and oriented along PA $=221^{\circ}$. The total on-source integration time was $2 \mathrm{~h}$, taken as six individual exposures of $1200 \mathrm{~s}$.

\footnotetext{
${ }^{1}$ IRAF is distributed by the National Optical Astronomy Observatory, which is operated by the Association of Universities for Research in Astronomy (AURA), Inc., under cooperative agreement with the National Science Foundation.
}

Data reduction was performed using the sub-packages of GEMINI.GMOS IRAF package and followed standard procedures including trimming of the images, bias subtraction, flat-fielding, wavelength calibration, sky subtraction, and relative flux calibration. We collapsed the individual data cubes in the spectral range of $\lambda \lambda 5500$ 6500 to create a GMOS/IFU continuum image similar to the HST F814W image.

Thereafter, we determined the centroid of each dithered image, and then spatially shifted and median combined the data cubes using the IRAF IMCOMBINE task with a SIGCLIP algorithm to eliminate the remaining cosmic rays and bad pixels. In order to suppress highfrequency noise in our data, we also applied the Butterworth filter ${ }^{2}$ of $0.2 \mathrm{Ny}$ set as upper limits of the pass-through frequency band to the data cube.

The final cube contains $\sim 7000$ spectra, each of which corresponds to a 'fiber' of dimensions 0 ' $1 \times 0$ ' 1 . The angular resolution of 0. ' 6 was derived from the full width at half-maximum (FWHM) of the spatial profile of the standard star. This resolution corresponds to a spatial scale of $\approx 300 \mathrm{pc}$ at the redshift of IRAS17526 +3253 .

In Fig. 1, the HST/ACS broad-band and continuum-subtracted $\mathrm{H} \alpha+[\mathrm{N} \mathrm{II}]$ images are compared with corresponding continuum and $\mathrm{H} \alpha$ images derived from the GMOS/IFU observations. The top left-hand and right-hand panels show, respectively, HST $i$-band and emission line images of the IRAS17526 + 3253 system. The middle panels show a region of $30 \operatorname{arcsec} \times 30$ arcsec centred at the NW nucleus, with the GMOS/IFU FOV being represented by a white box. The bottom panels show the HST and corresponding GMOS/IFU images within the IFU FOV. The GMOS/IFU continuum image was constructed by collapsing the data cube along the spectral axis between 5500 and $6500 \AA$. The $\mathrm{H} \alpha$ image was obtained by fitting the emission line profiles in each spaxel (see Section 3.2). The HST images were convolved by a Gaussian function with sigma equal to 5 pixels $(\sim 0.25$ arcsec) and spatial registration was carried out by cross-matching these images with the corresponding IFU images in order to make the spatial resolution closer to that of the GMOS/IFU data. Throughout this paper, we define the origin of the spatial scale to be that of the spaxel in the GMOS/IFU $\mathrm{H} \alpha$ FOV corresponding to the peak surface brightness of the brightest knot (bottom left in rightmost bottom panel of Fig. 1), whose coordinates are RA: $17^{\mathrm{h}} 54^{\mathrm{m}} 29.4^{\mathrm{s}}$ and Dec: $+32^{\mathrm{d}} 53^{\mathrm{m}} 12.8^{\mathrm{s}}$

\section{RESULTS}

\subsection{Optical to near-IR morphologies}

The HST/ACS images in the top row of Fig. 1 clearly reveal a merger system in mid-stage with two main galaxy nuclei separated by a projected distance of $\sim 8.8 \mathrm{kpc}$. The NW galaxy appears to present a nearly edge-on aspect, and is crossed by a prominent dust lane, suggesting that the northern side is the near side. The disrupted disc of the second galaxy (Eastern nucleus) of the interacting pair appears to the merging with that of its companion and features a complex system of dust lanes.

The two nuclei are embedded in an elongated irregular envelope, which is interspersed with extensive dust lanes and extends over at least $60 \operatorname{arcsec}(30 \mathrm{kpc})$, with its major axis oriented approximately NW-SE. The elongation suggests that we are observing the interaction quite close to the orbital plane. The $\mathrm{H} \alpha+[\mathrm{N}$ II $]$ emission image, which is a tracer of young stars via $\mathrm{H}$ II regions, shows numerous

\footnotetext{
${ }^{2} \mathrm{http} / / /$ www.exelisvis.com/docs/BANDPASSFILTER.html
} 

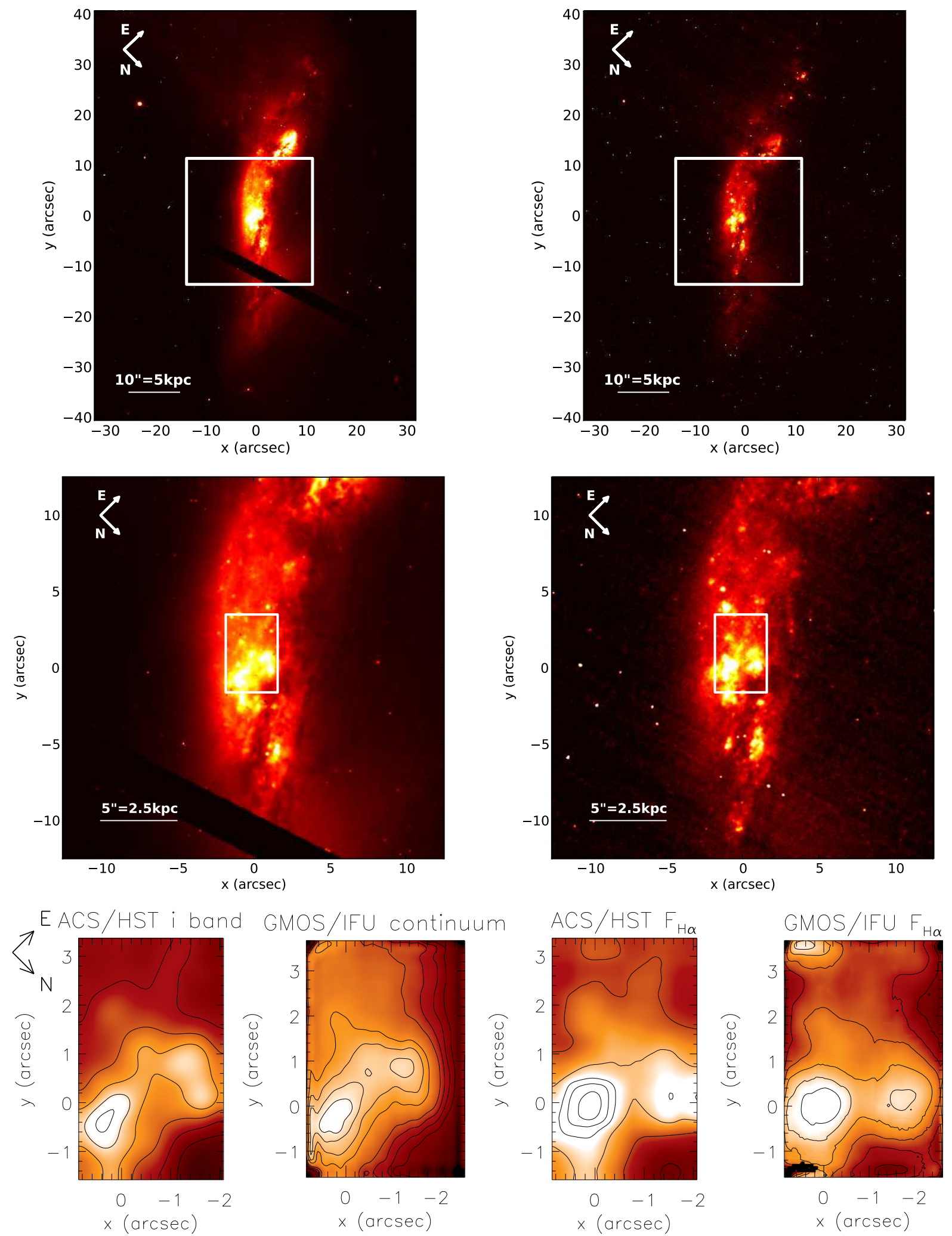

Figure 1. HST/ACS and reconstructed GMOS/IFU images of IRAS17526 + 3253. Top left: large-scale HST/ACS F814W ( $i$ band) image. Top right: large-scale continuum free $\mathrm{H} \alpha+[\mathrm{N} \mathrm{II}]$ image derived from HST/ACS FR656N and FR914M ramp filter images. The boxes represent the FOV shown in the middle panels. Middle left: medium-scale image from ACS/HST F814W centred on the NW nucleus that was observed with GMOS/IFU. Middle right: the same for the HST $\mathrm{H} \alpha+[\mathrm{N}$ II] image. The boxes represent the GMOS/IFU FOV. Bottom panels show images of the GMOS/IFU FOV: from left to right, HST/ACS F814W $i$ band, GMOS/IFU continuum ( $\lambda \lambda$ 5500-6500), continuum free HST $\mathrm{H} \alpha+[\mathrm{N}$ II], $\mathrm{H} \alpha$ flux map reconstructed from the GMOS/IFU spectroscopy. 


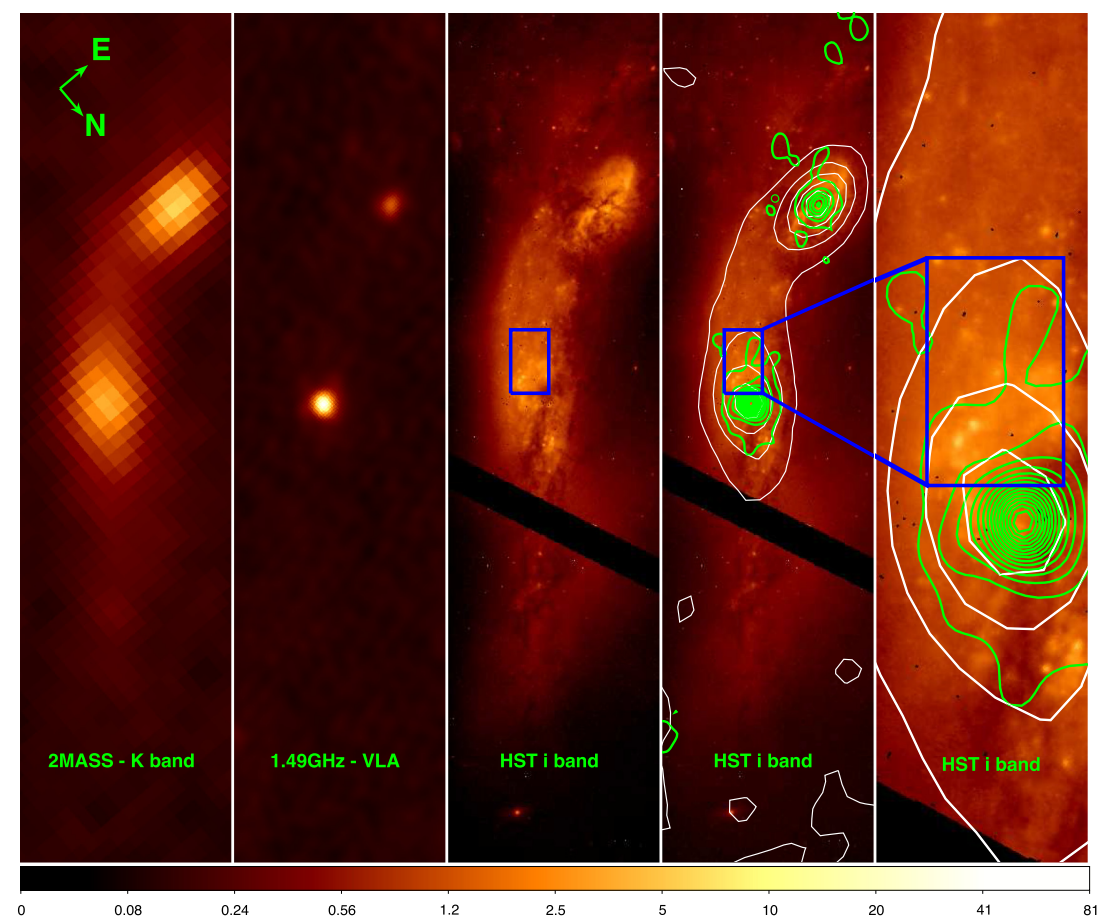

Figure 2. Comparison of NIR, radio, and optical morphology. From left to right: 2MASS $K$-band image; 1.49 GHz VLA image; $H S T / A C S$ F814W image, with IFU field shown in blue; $H S T / A C S$ F814W image with $1.49 \mathrm{GHz}$ radio and $K$-band contours overplotted in green and white, respectively; zoomed in view of the NW nucleus in the HST $i$-band image. Scale bar is in arbitrary units.

compact regions of ongoing star formation across the envelope over scales of a few tens of kpc. Baan \& Klöckner (2006) have shown that the radio emission of these smaller knots in IRAS17526 + 3253 is probably associated with signal-to-noise ratios (SNRs) in massive star-forming regions.

The middle panels of Fig. 1 show a region of $30 \operatorname{arcsec} \times$ 30 arcsec centred on the NW nucleus with the GMOS/IFU FOV being represented by a white box. The HST/ACS $i$-band continuum image shows a very disturbed morphology with two bright 'blobs' within the IFU field. These features are also prominent in the $\mathrm{H} \alpha$ $+[\mathrm{N}$ II $]$ image, indicating that they are star-forming regions. The IFU field was placed to sample these bright star-forming regions, and it is located just south of the extensive wide dust lane crossing the nucleus; the dust lane can be seen in both the $i$-band and $\mathrm{H} \alpha+[\mathrm{N}$ II] images and crosses the NW corner of the IFU field. In the bottom row of Fig. 1, the seeing-convolved HST $i$ band and $\mathrm{H} \alpha+[\mathrm{N}$ II] images are compared with corresponding images reconstructed from our GMOS/IFU data. The morphological features appearing in the IFU images match those seen in the HST well and in particular, the two bright blobs are clearly resolved in the IFU data.

The optical (HST/ACS F814W), near-IR (NIR; 2MASS $K$ band), and radio (VLA $1.49 \mathrm{GHz}$ ) morphologies of the whole IRAS17526 + 3253 system are compared in Fig. 2 . The $K$-band image is less affected by dust extinction and highlights the old stellar population, which can be used as an indication of stellar mass distribution of the compact central cores. The overall morphology in the NIR is similar to that seen at much higher resolution in the $i$ band, although the two nuclei are much more prominent relative to the surrounding envelope. From $K s$ band photometry, we find that the two nuclei have very similar fluxes, $\sim 12.1 \mathrm{mag}$, which translates to a bulge mass of $\sim 5.3 \times 10^{9} \mathrm{M}_{\odot}$, using the scale factor inferred by Bell et al. (2003). We also modelled the surface brightness distribution of the 2MASS $K$-band image using GALFIT (Peng et al. 2002). It turns out that both nuclei have similar Sérsic indices, indicative of radial brightness profiles consistent with classical bulges $\left(\mathrm{n}_{s} \approx 2.2\right)$. The main features in the VLA $1.49 \mathrm{GHz}$ image are two bright, compact cores (beam size of $1^{\prime \prime} .35 \times 1^{\prime \prime} .11$ ) that are clearly associated with the two galaxy nuclei, as shown by the contours overplotted on the $H S T i$-band image in the leftmost two panels.

\subsection{GMOS/IFU data analysis: emission line fitting and kinematics}

This section describes the techniques employed to derive quantitative information from the GMOS/IFU data cube. To accomplish this, we used customized PROFIT routines written in the $\mathrm{IDL}^{3}$ programming language. More information about the PROFIT code can be found in Riffel (2010).

\subsubsection{Single component Gauss-Hermite fits}

The set of spectra in the GMOS/IFU data cube presents narrow emission lines, with an average FWHM of $\sim 5 \AA$. The profiles of emission lines clearly change over the FOV that various regions exhibit profiles with different characteristics: (i) narrow emission lines displaying a single peak, (ii) emission lines characterized by a single peak with asymmetric extended wings due to blueshifted or redshifted components, and (iii) line profiles showing a clear double peak.

We present in Fig. 3 spectra extracted from spaxels $\left(0{ }^{\prime} .1 \times 00^{\prime} .1\right)$ at six locations within the GMOS/IFU FOV, including the peak flux positions of the two bright blobs, labelled as (a) and (b) in the figure (top right-hand panels). The other four extractions (labelled as c, $\mathrm{d}$, e, and $\mathrm{f}$ ) were selected to illustrate the different emission line

\footnotetext{
${ }^{3}$ Interactive Data Language, http://ittvis.com/idl.
} 

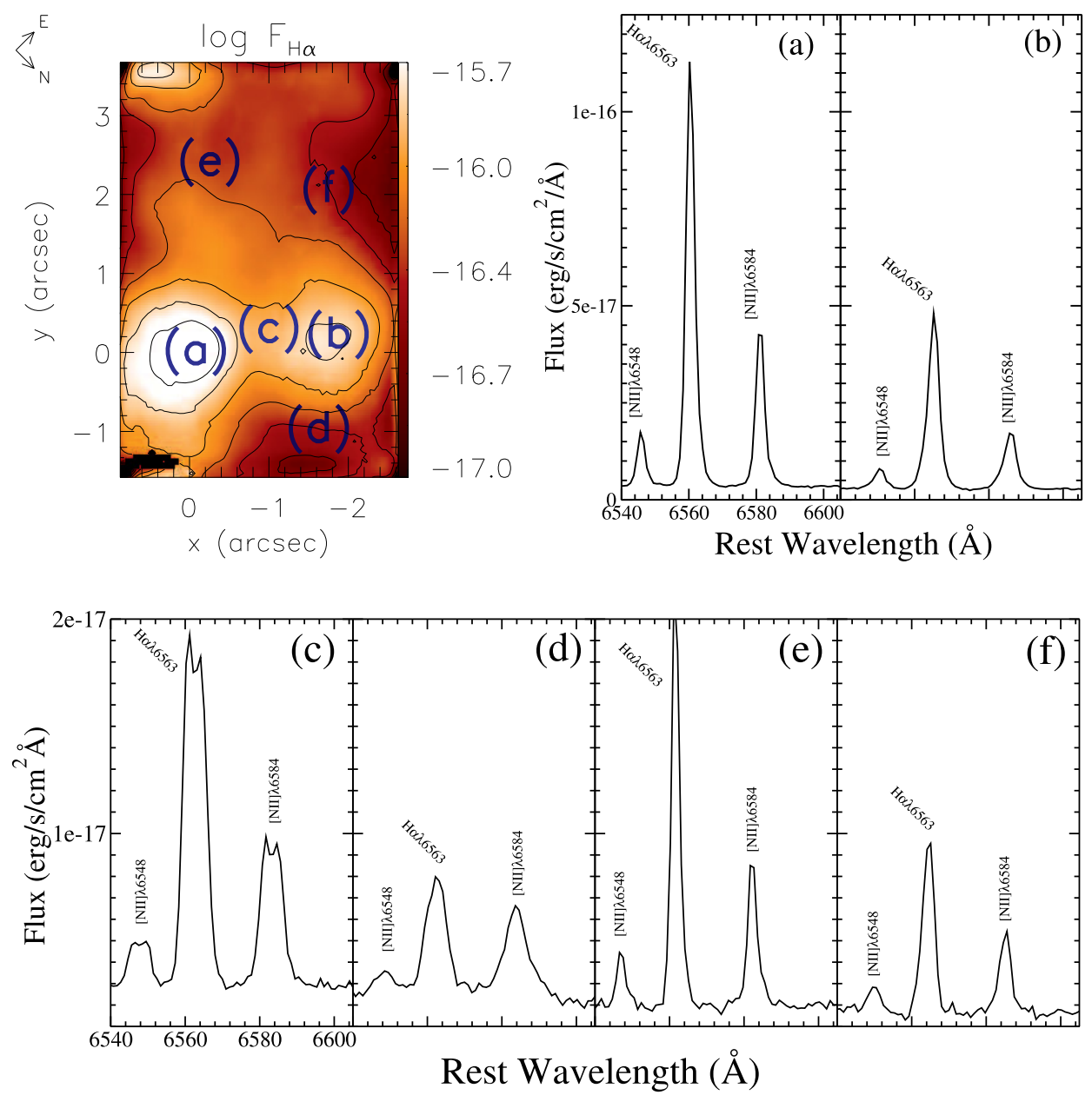

Figure 3. Top left-hand panel: emission-line map of $\mathrm{H} \alpha$ reconstructed from the GMOS/IFU spectroscopy and flux unit are shown in logarithmic scale of $\mathrm{erg} \mathrm{s}^{-1} \mathrm{~cm}^{2}$ fiber ${ }^{-1}$. Top right-hand panel: spectra corresponding to left and right blobs seem in the $\mathrm{H} \alpha$ image. Bottom panels: sample of spectra extracted at the positions (c), (d), (e), and (f) labelled in the $\mathrm{H} \alpha$ flux map.

profiles and excitation conditions that are present within the FOV. In the spectra from regions (e) and (f) as well as those from the blobs (a) and (b), $\mathrm{H} \alpha$ is much stronger than $[\mathrm{N}$ II] $]$ 6583 and the profiles are relatively narrow. In region (c), which is located between the two blobs, the lines are broader but double peaked, suggesting partial blending of two components associated with blobs (a) and (b), which present a discontinuity in velocity, as discussed next. Region (d) on the other hand, exhibits much broader profiles and $\mathrm{H} \alpha$ is only slightly stronger than $[\mathrm{N} \mathrm{II}] \lambda 6583$.

Fig. 4 shows the entire spectra, covering the whole observed wavelength range, of the blobs (a) and (b). In addition to $\mathrm{H} \alpha$ and the $[\mathrm{N}$ II] $] \lambda 6548,83$ lines, $\mathrm{H} \beta,[\mathrm{O}$ III] $\lambda 4959,5007$, [O I] $\lambda 6300$ and $[\mathrm{S}$ II] $] \lambda 6717,6731$ are also present in the spectra. The relative strengths of the lines are consistent with stellar-photoionized H II regions.

As the emission line profiles are clearly non-Gaussian in certain regions, we used a Gauss-Hermite function to perform single component fits to each emission lines. The Gauss-Hermite series can be written as

$\mathcal{G H}=\Psi \frac{\alpha(w)}{\sigma}\left[1+h_{3} \mathcal{H}_{3}(w)+h_{4} \mathcal{H}_{4}(w)\right]$

where $w \equiv\left(\lambda-\lambda_{c}\right) / \sigma$ and $\alpha(w)=(1 / \sqrt{2 \pi}) e^{w^{2} / 2}$.
$\Psi$ represents the amplitude of the Gauss-Hermite series, $\lambda_{c}$ is the central wavelength, $h_{3}$ and $h_{4}$ are moments of Gauss-Hermite series, which measure, respectively, asymmetric and symmetric deviations from a pure Gaussian. $\mathcal{H}_{3}(w)$ and $\mathcal{H}_{4}(w)$ are Hermite polynomials. In order to fit the $\mathrm{H} \alpha+[\mathrm{N} \mathrm{II}]$ lines, we assumed that $\mathrm{H} \alpha$ and [N II] have the same central velocity, while the amplitude of $[\mathrm{N} \mathrm{II]}] \lambda 6548$ component was constrained to be $1 / 2.96$ of that of $[\mathrm{N} \mathrm{II}] \lambda 6583$. Similar constrains were applied to fit the $[\mathrm{O}$ III $] \lambda \lambda 4959,5007$ lines. The $[\mathrm{S}$ II] $] \lambda \lambda 6717,6731$ lines were constrained to have the same central velocity.

The results of the emission line fitting are shown in Fig. 5. The values of $h_{3}$ and $h_{4}$ vary from -0.2 to 0.2 . Visual inspection of the fits suggests that emission lines with blue wings show values of $h_{3}<-0.1$, while the red wings appear in regions with $h_{3}>$ 0.1 . Values of $h_{4}<-0.1$ select regions where the line profiles are broader (not as sharply peaked) than an equivalent Gaussian of the same amplitude, including profiles that have noticeable double peaks. The values of $h_{3}$ and $h_{4}$ recovered from the fits therefore allow extended asymmetric wings and double-peaked profiles to be automatically identified. We used these values to generate a mask that flags spaxels that required more than one Gaussian to fit the observed emission lines. The mask image is also shown in Fig. 5. 


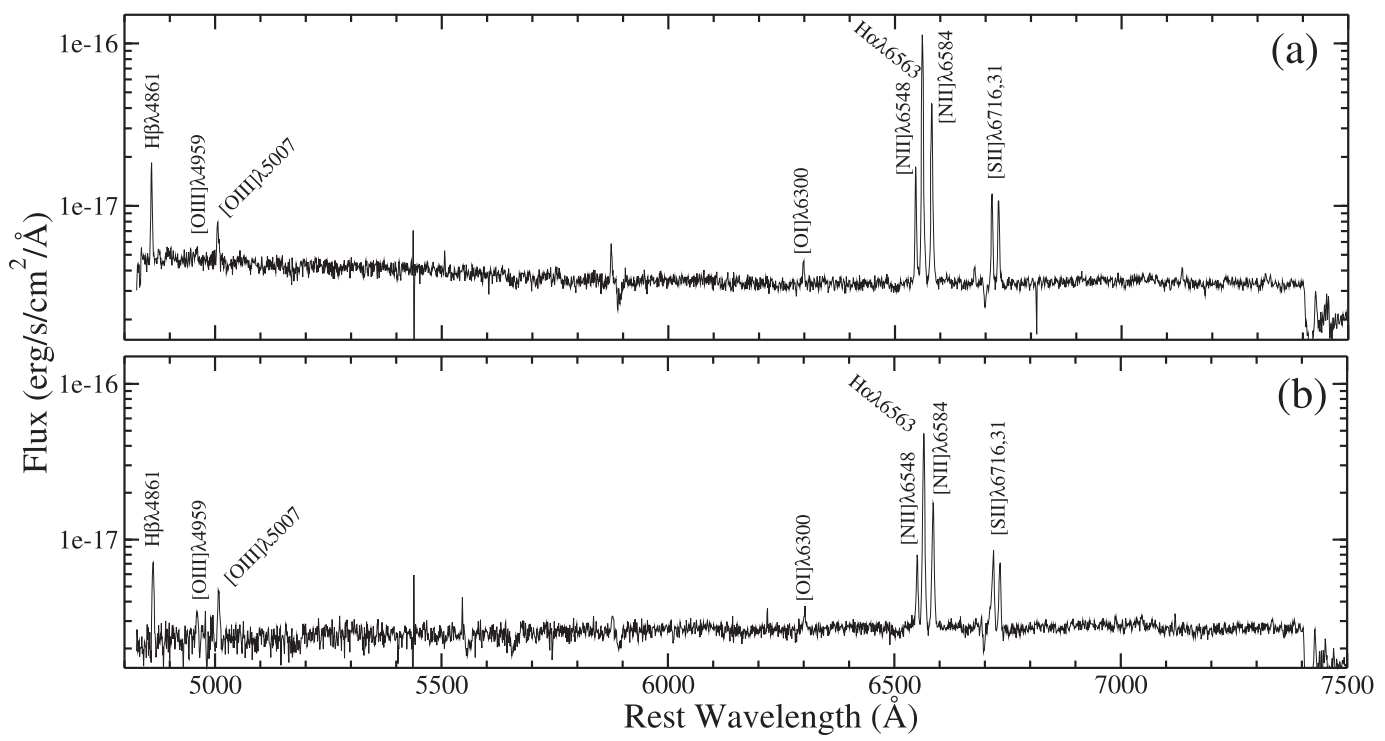

Figure 4. The whole observed GMOS/IFU spectral range of $\mathrm{NW}_{a}$ and $\mathrm{NW}_{b}$. The emission lines are labelled.
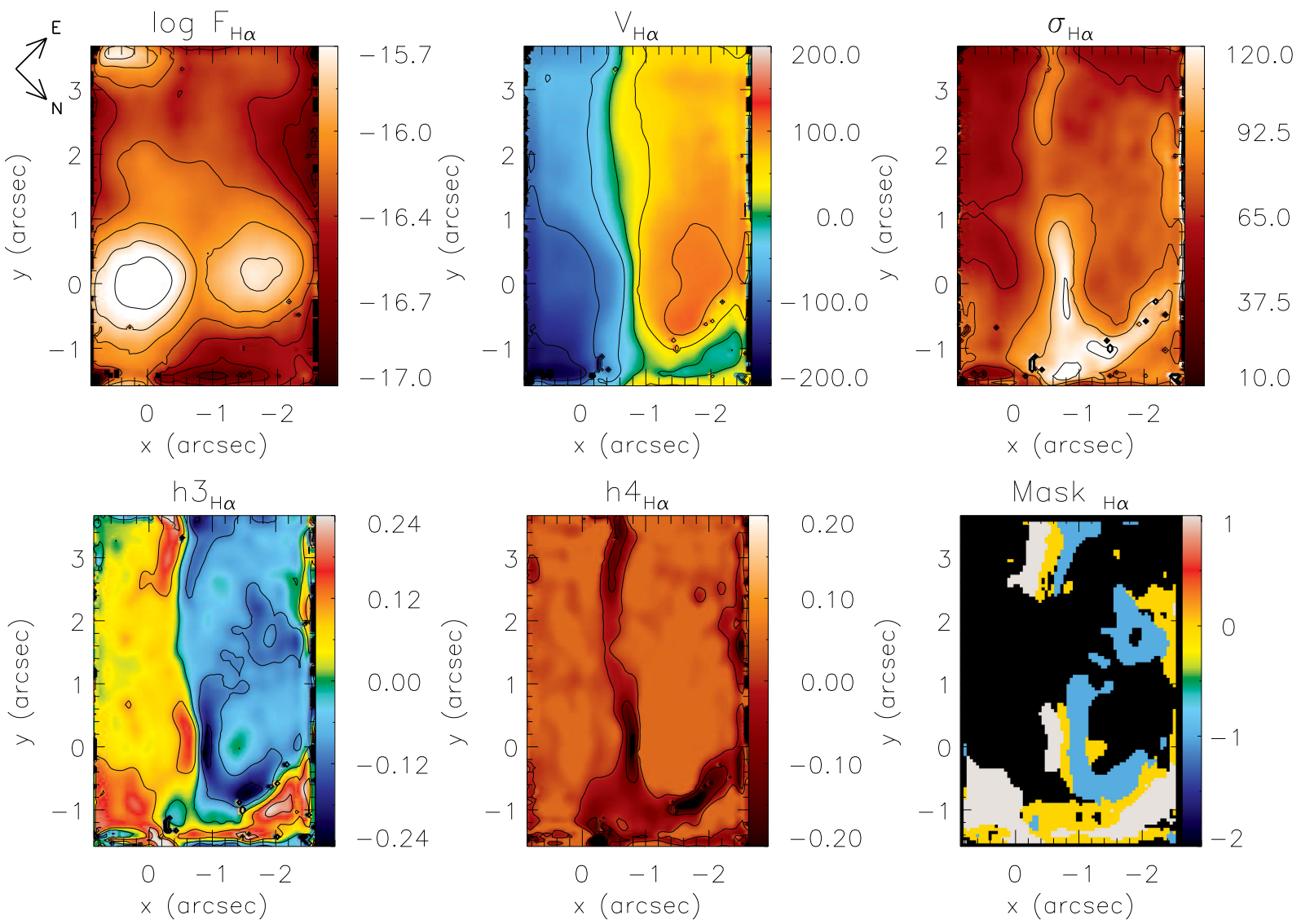

Figure 5. Results from Gauss-Hermite fits of $\mathrm{H} \alpha$ emission line. Top panels show flux emission maps (left-hand panel), velocity field (middle panel), and velocity dispersion (right-hand panel) maps. Bottom left-hand and middle panels show Gauss-Hermite $\mathrm{h}_{3}$ and $\mathrm{h}_{4}$ moments. Bottom right-hand panel presents masked regions that received values from two Gaussian fits. Flux units are shown in logarithmic scale of $\mathrm{erg} \mathrm{s}^{-1} \mathrm{~cm}^{2} \mathrm{fiber}^{-1}$.

The $\mathrm{H} \alpha$ flux map obtained by Gauss-Hermite fitting highlights the presence of the two bright blobs that are the most prominent features in the IFU FOV, as already seen in the HST $i$-band and $\mathrm{H} \alpha$ $+[\mathrm{N} \mathrm{II}]$ emission line images (see Fig. 1). A smaller and somewhat fainter third blob is located near the southern corner of the FOV, which appears to be associated with a bright knot seen in the HST $\mathrm{H} \alpha+[\mathrm{N} \mathrm{II}]$ image just beyond the SE border of the IFU field (see Fig. 1). The flux distributions of the other emission lines (with the 
exception of $[\mathrm{O} I] \lambda \lambda 6300)$ show features similar to those observed in the $\mathrm{H} \alpha$ map, but with lower SNR, and thus we do not present them in this section.

It is immediately clear from visual inspection of the data cube that the emission lines of the brightest blob (NW, hereafter $\mathrm{NW}_{a}$ ) are blueshifted with respect to the line emission at the centre of GMOS/IFU FOV, while the northeastern blob (hereafter $\mathrm{NW}_{b}$ ) appears to have redshifted emission lines. We therefore determined the systemic velocity for the IFU field by averaging the velocities of two bright blobs $\left(v_{\text {sys }}=7580 \mathrm{~km} \mathrm{~s}^{-1}\right)$ within apertures of $0.75 \mathrm{arcsec}$ centred on the flux peaks. Maps of the centroid velocity, corrected for this systemic velocity, and the velocity dispersion, corrected for the instrumental resolution, are shown in Fig. 5.

The velocity map shows blueshifts dominating the SW side of the FOV, while redshifts dominate the NE side. These regions are very clearly segregated, with a sharp transition between the blue and redshifted sides. This velocity field does not show the 'spider' diagram typical of disc rotation. Moreover, given the morphology and apparent orientation of the NW galaxy, we would expect the position angle of the line of nodes to be approximately NW-SE, nearly orthogonal to the direction one would infer from the velocity map. It is also notable that the two regions, or the boundary between them, are clearly distinguishable in the $\sigma, h_{3}$, and $h_{4}$ maps. Thus, $h_{3}$ shows that the emission lines in the SW (blueshifted) region are characterized by red asymmetries, whereas in the NE (redshifted) region the lines are typically blue asymmetric. The $\sigma$ and $h_{4}$ maps both show narrow features that coincide with the sharp boundary between the blue and redshifted regions, indicating that the lines are broader and more flat topped along the boundary. Indeed, this is where we observe double-peaked profiles as exemplified by the region (d) spectrum shown in Fig. 3. Taken together, the kinematic parameter maps suggest that we are observing two distinct, partially superimposed kinematic components that have a relative velocity $\sim 200 \mathrm{~km} \mathrm{~s}^{-1}$.

There is also evidence for a third kinematic component, which is traced by the region of increased velocity dispersion close to the NW edge of the FOV, with a spur running NE. Similar structures are seen in the $h_{3}$ and $h_{4}$ maps, indicating red asymmetric, broader lines.

\subsubsection{Two-component Gaussian fits}

As described above, the results of the single-component GaussHermite fits have been used to identify spaxels where the emission lines cannot be well fitted with a single Gaussian profile (see Fig. 5). These include spaxels where the lines have a single peak but extended or asymmetric wings, as well as those where the lines exhibit double peaks. As these profile shapes may result from blending of kinematic components arising from distinct kinematic components, we have used two Gaussian components to fit the line profiles in those spaxels that have $-0.1<h_{3}<0.1$ and $h_{4}$ $<-0.1$. The first criteria track spaxels that have emission line with extended wings, once that second one identifies spaxels with double peaks of the emission profile. These regions are indicated with values between -1 and 1 in the mask image shown in Fig. 5 . A single Gaussian component was used to fit the line profiles over the remainder of the FoV. The same constraints as used in the Gauss-Hermite fits (Section 3.2.1) were applied to each Gaussian component in the fits to the $\mathrm{H} \alpha+[\mathrm{N}$ II], [O III] $\lambda \lambda 44959,5007$ and $[\mathrm{S}$ II] $\lambda \lambda 6717,6731$ line groups.
The flux distribution, velocity, and velocity dispersion (after correction for instrumental resolution) maps derived from the single- and double-Gaussian fits are presented for the $\mathrm{H} \alpha$ emission line in Fig. 6. It can be seen from the flux distributions that the region in which single Gaussian fits were used includes the two prominent flux peaks previously identified as $\mathrm{NW}_{a}$ and $\mathrm{NW}_{b}$, whereas the regions where the profiles exhibit splitting or extended wings and where two Gaussian components were used, mainly lie between the blobs, or border them along the NW edge of the FOV.

Not surprisingly, the velocity field within the region where the lines were fitted with a single Gaussian component is very similar to that obtained from the single Gauss-Hermite series, exhibiting the same pattern of blueshifts on the SW side of the FOV, and redshifts on the NE side. The velocities obtained from the blue and redshifted components of the two-component Gaussian fits are generally comparable with those obtained from the singlecomponent fits in the blueshifted and redshifted sides of the FOV. The main exception is a region close to the NW border of the FOV, where the red component has a much higher velocity $\left(\sim 200 \mathrm{~km} \mathrm{~s}^{-1}\right)$ than is seen elsewhere. This location also coincides with a high velocity dispersion in both the red and blue components. However, the blue component in this region does not show a correspondingly large blueshift, and its velocity in fact is quite comparable with the blueshifted velocities over the SW side of the FOV.

In Fig. 7, we present velocity channel maps extracted along the $\mathrm{H} \alpha$ line profile, using velocity bins of $45 \mathrm{~km} \mathrm{~s}^{-1}$. The distribution of $\mathrm{H} \alpha$ emission in these velocity slices also suggests that there are two distinct but partially overlapping kinematic components. Approximately half of the FOV on the SW side is covered by predominately blueshifted emission that includes the prominent flux peak, $\mathrm{NW}_{a}$, as well as the fainter blob at the south corner of the FOV, which appears to be connected to $\mathrm{NW}_{a}$ by a ridge of emission. These structures can be seen at velocities ranging from $\sim-200$ to $+70 \mathrm{~km}$ $\mathrm{s}^{-1}$ at $\mathrm{NW}_{a}$. The NE half of the FOV has dominated the redshifted emission. The main feature is $\mathrm{NW}_{b}$, but there is also a ridge of emission extending to the SE, with these structures spanning the velocity range $\sim 70-300 \mathrm{~km} \mathrm{~s}^{-1}$.

To gain further insights into the correlations between the spatial distribution of the line-emitting gas and its velocity field, we applied principal components analysis (PCA) to the IFU data cube. PCA is a statistical technique that has recently found various applications in astrophysics and in particular has been extensively applied to analyse IFU observations (PCA, e.g. Murtagh \& Heck 1987; Steiner et al. 2009; Ricci, Steiner \& Menezes 2011). This tool transforms the system of correlated coordinates into a system of orthogonal coordinates, and eigenvectors, ordered by principal components of decreasing variance. The projection of the data on to these coordinates produces images called tomograms, while eigenvectors correspond to the eigenspectra. The combined analysis of both can be used to identify distinct components in the IFU data, which facilitates physical interpretation.

The results of our PCA analysis of the IRAS17526 + 3253 IFU data are shown in Fig. 8. The derived tomograms and their respective eigenspectra are shown in the left-hand and right-hand columns, respectively. The first eigenspectrum, which accounts for $\sim 74$ per cent of the data cube variance, corresponds to the blueshifted emission and the bright $\mathrm{NW}_{a}$ in the SW side of the FOV. The second, which accounts for $\sim 16$ per cent of the variance, is mainly associated with the redshifted emission including $\mathrm{NW}_{b}$, a weaker structure extending in the direction of $\mathrm{NW}_{a}$ and a diffuse feature extending to the SE. The third and fourth eigenspectra account for the remaining $\sim 8$ per cent of the variance in the data cube 

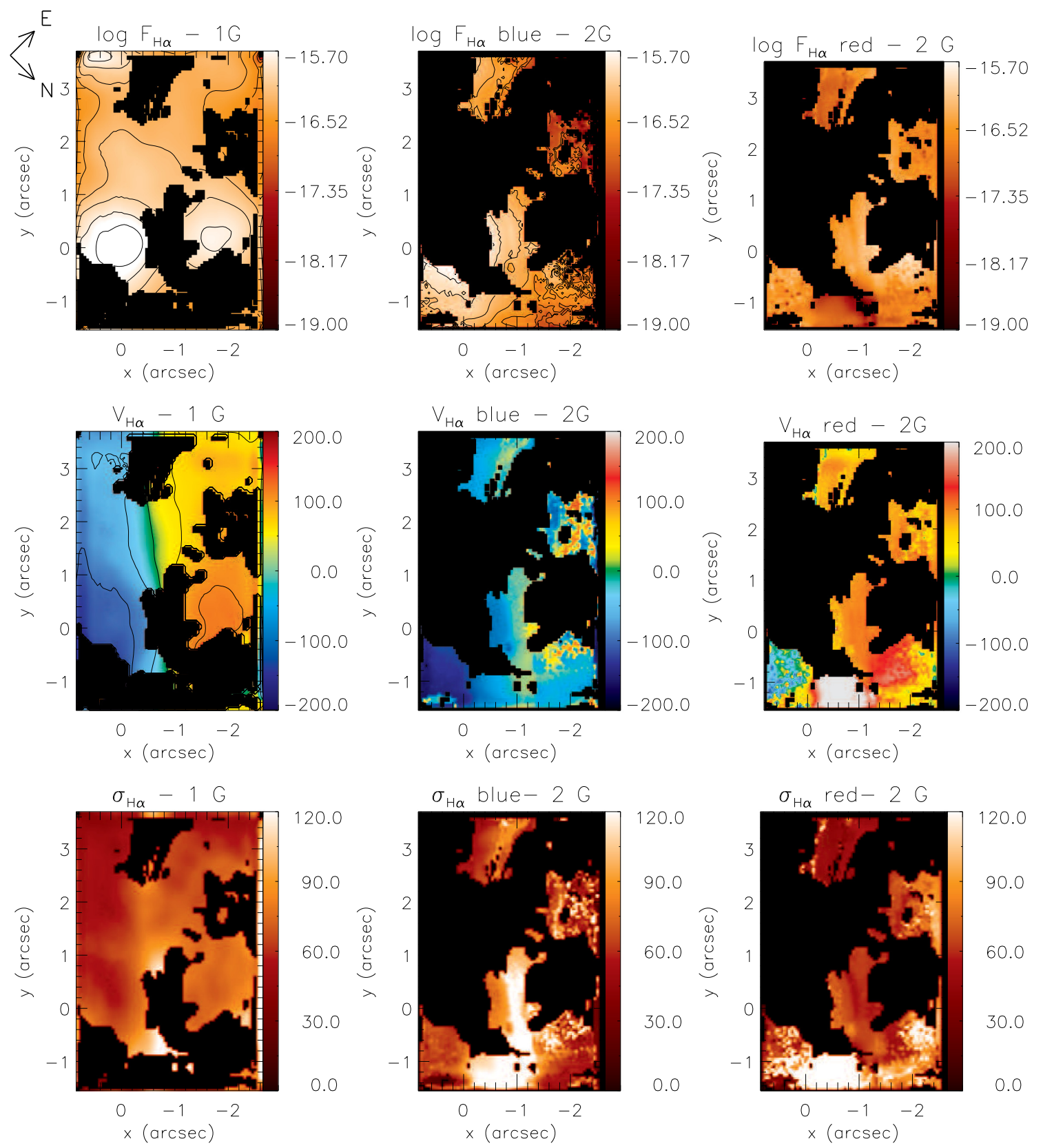

Figure 6. Top-panels: emission line maps of $\mathrm{H} \alpha$. Top left-hand panel is emission flux taken from one Gaussian fitting. Middle and top right-panels are, respectively, the blueshifted and redshifted components derived from two Gaussian fitting of $\mathrm{H} \alpha$ emission. Middle panels are the same for velocity maps. Velocity dispersion are showed in the bottom panels. Flux units are shown in logarithmic scale of $\mathrm{erg} \mathrm{s}^{-1} \mathrm{~cm}^{2} \mathrm{fiber}^{-1}$.

and exhibit features associated, for example, with the peak of $\mathrm{NW}_{a}$, the knot at the $\mathrm{S}$ corner of the FOV and the boundary between the redshifted and blueshifted regions. Overall, the structures revealed by the PCA analysis exhibit a very close correspondence with the features seen in the velocity channel maps.

Together with the channel maps, the results of the PCA analysis support the interpretation of the parameter maps derived from line fitting that the SW and NE sides of the FOV sample kinematically independent systems, which are, respectively, blueshifted and redshifted relative to the adopted systemic velocity. Accordingly, we have constructed separate maps of these regions for selected emission lines by combining the velocities, velocity dispersions, and fluxes from the single-component
Gaussian fit with the corresponding values for the blueshifted and redshifted components, respectively, of the double Gaussian fits.

The flux maps for the $\mathrm{H} \beta$, [O III] $\lambda \lambda 5007$, [O I ] $\lambda \lambda 6300$, $[\mathrm{N}$ II] $] \lambda \lambda 6584, \mathrm{H} \alpha$, and $[\mathrm{S} \mathrm{II}] \lambda \lambda 6731$ emission lines are presented for the blueshifted region in Fig. 9. With the exception of [O I] $\lambda \lambda 6300$, the line fluxes show similar distributions, the main feature being $\mathrm{NW}_{a}$ with an angular size of roughly $0^{\prime \prime} .75(\approx 400 \mathrm{pc})$. The fainter blob some 3.5 to the SE is also seen in all lines, except for [O III] $\lambda \lambda 5007$ (probably due to the lower SNR). The [O I] 6300 line is relatively weak over most of the FOV $(\mathrm{SNR} \approx 5)$, but it is notable that its flux peak occurs near the NW edge of the field, offset by about 1 ". 5 to the North of $\mathrm{NW}_{a}$. 

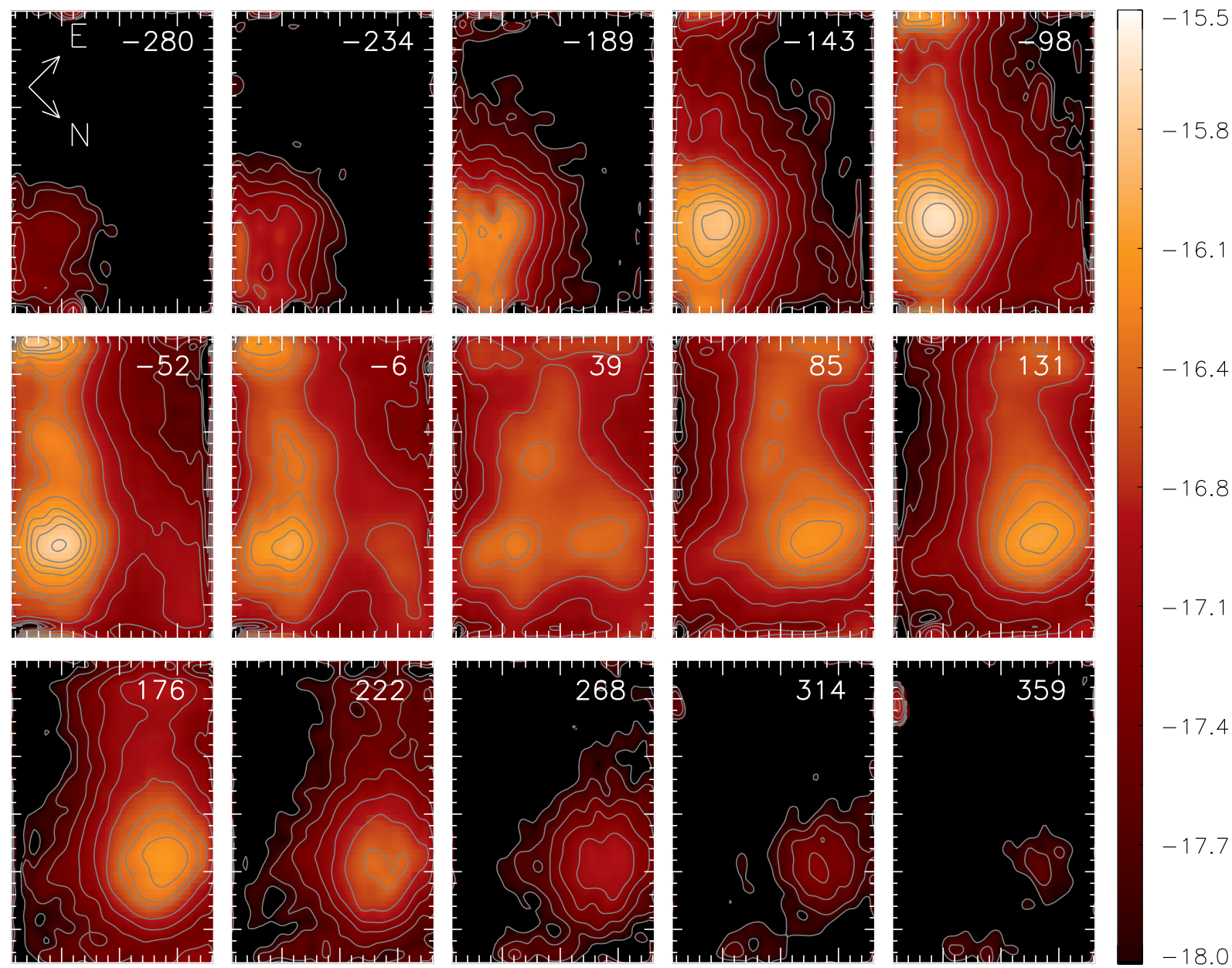

Figure 7. Observed radial velocity channel maps of the $\mathrm{H} \alpha$ emission line are plotted with a logarithmic scale. The radial velocity range from -337 to $+303 \mathrm{~km} \mathrm{~s}^{-1}$ in a steps of $46 \mathrm{~km} \mathrm{~s}^{-1}$. The velocity of each channel is shown in the top right-hand corner in the corresponding panel. The intensity scale is displayed at the right side.

Fig. 10 shows the centroid velocity (left-hand panels) and velocity dispersion (right-hand panels) maps for the $\mathrm{H} \alpha$, [O III] $] \lambda 25007$ and $[\mathrm{S}$ II] $] \lambda \lambda 6716$ lines in the blueshifted region, after subtraction of the systemic velocity $\left(v_{\text {sys }}=7580 \mathrm{~km} \mathrm{~s}^{-1}\right)$ and correction for instrumental resolution, respectively. As the corresponding maps for $[\mathrm{N}$ II] $] \lambda 6584$ are similar to those of $\mathrm{H} \alpha$ line, they are not shown. As a whole, the gas in the southwestern half of the FOV exhibits a NW-SE velocity gradient, with the largest blueshifts $(\sim-150 \mathrm{~km}$ $\mathrm{s}^{-1}$ ) occurring in a region just south of $\mathrm{NW}_{a}$. Blob $\mathrm{NW}_{a}$ itself has a velocity $\left(\sim-100 \mathrm{~km} \mathrm{~s}^{-1}\right)$, while the diffuse emission has velocities $\left(\sim-50 \mathrm{~km} \mathrm{~s}^{-1}\right)$. The highest velocity dispersions $\left(\gtrsim 100 \mathrm{~km} \mathrm{~s}^{-1}\right)$ occur in a localized region near the NW border of the FOV, and roughly centred between the two bright blobs. A ridge of higher velocity dispersion also extends SE along the border between the blueshifted and redshifted regions. However, outside these localized enhancements, the velocity dispersion is fairly uniform for the remainder of the blueshifted region, with a typical value $\sim 50 \mathrm{~km}$ $\mathrm{s}^{-1}$ (the small-scale structure in the [O III] $\lambda 5007$ map is due to the low SNR). In particular, neither $\mathrm{NW}_{a}$ or the secondary knot at the $\mathrm{S}$ corner of the FOV correspond to obvious features in the velocity dispersion map.
The emission-line flux, centroid velocity, and velocity dispersion maps for the redshifted region are presented in Figs 11 and 12. The main emission component in most lines is the component identified as $\mathrm{NW}_{b}$, which has a similar angular size to that of $\mathrm{NW}_{a}\left(0^{\prime \prime} \cdot 75\right.$, and $\approx 400 \mathrm{pc}$ ). However, just as in the corresponding flux map for the blueshifted region (Fig. 11), the strongest [O I] 6300 emission is located close to the NW edge of the FOV, bordering $\mathrm{NW}_{a}$ to the $\mathrm{N}$. To a lesser extent, this feature is also seen in [N II] $] \lambda 26584$.

The velocity field of the redshifted region shows a similar gradient to that of the blueshifted region (12): the highest velocities $(\sim+$ $150 \mathrm{~km} \mathrm{~s}^{-1}$ ) occur just $\mathrm{NW}$ of $\mathrm{NW}_{a}$, which itself has a velocity $\sim+100 \mathrm{~km} \mathrm{~s}^{-1}$, whereas the diffuse emission to the SE has a typical velocity $\sim+50 \mathrm{~km} \mathrm{~s}^{-1}$. However, there is also a distinct region, spatially coincident with the region where the $[\mathrm{O}$ I $] \lambda 6300$ emission peaks, which clearly exhibits a high velocity $(\sim+200 \mathrm{~km}$ $\left.\mathrm{s}^{-1}\right)$ and velocity dispersion $\left(\sim 150 \mathrm{~km} \mathrm{~s}^{-1}\right)$ in $\mathrm{H} \alpha$, and possibly the other lines. The velocity dispersion is also generally enhanced $\left(\sim+200 \mathrm{~km} \mathrm{~s}^{-1}\right)$ between the NW edge of the FOV and blobs $\mathrm{NW}_{a}$ and $\mathrm{NW}_{b}$. It is worth noting that this side of the IFU FOV borders the radio source associated with the nucleus of the NW galaxy (see Fig. 2). Over the rest of the redshifted region, including $\mathrm{NW}_{b}$ and the 

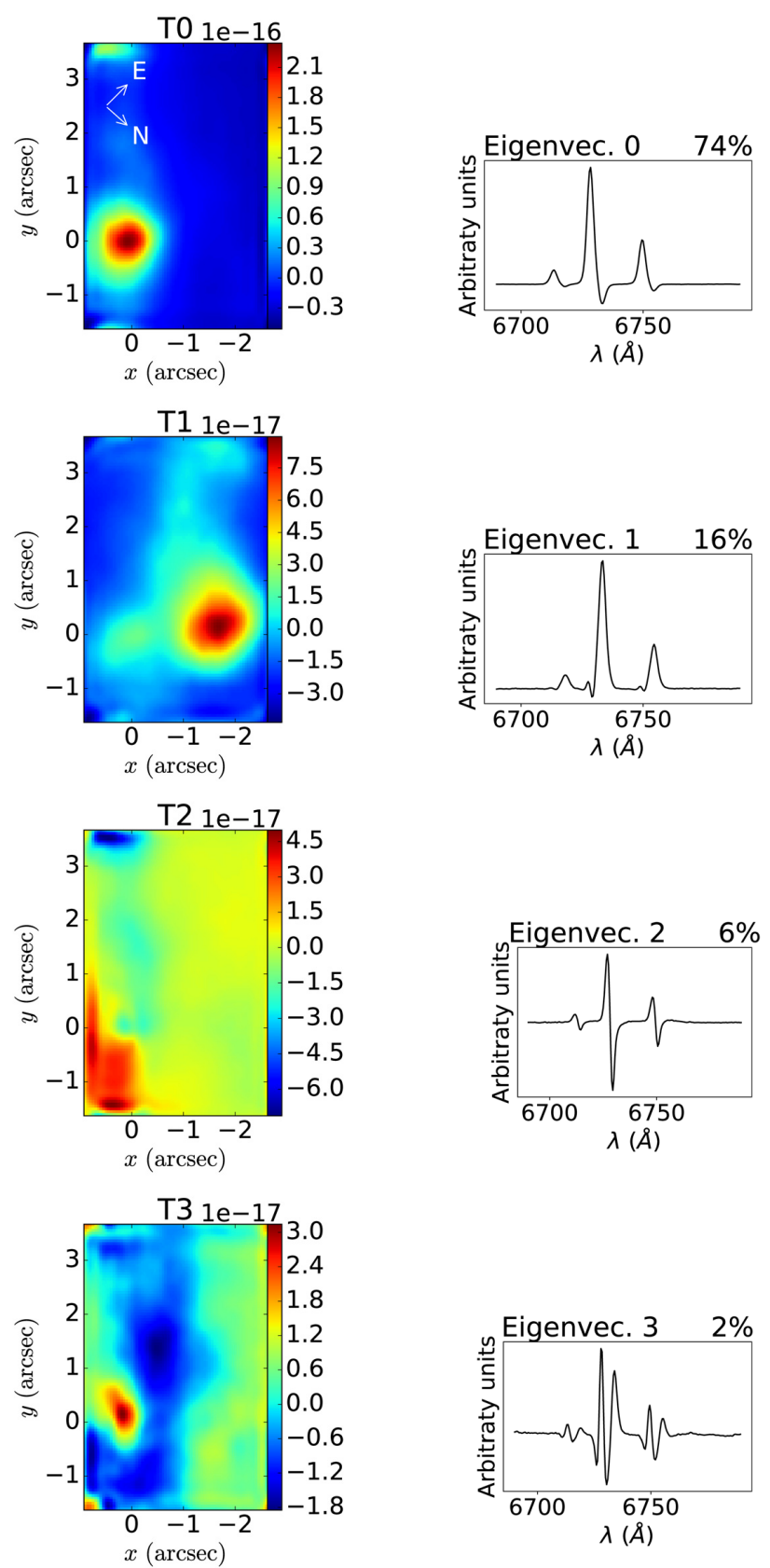

Figure 8. PCA derived from GMOS/IFU data. From top to bottom: tomograms (left-hand panels) and eigenspectra (right-hand panels) corresponding to first four principal components of the data cube of IRAS17526 + 3253 .

diffuse emission extending SE, the velocity dispersion is typically relatively low $\left(\sim 50 \mathrm{~km} \mathrm{~s}^{-1}\right)$.

\subsection{GMOS/IFU data analysis: emission line ratios and ionization}

In order to investigate the ionization mechanisms responsible for the line emission in the region sampled by our IFU observations, we have used the line fluxes derived from the Gaussian fits to construct emission line ratio diagnostic BPT diagrams (Baldwin, Phillips \& Terlevich 1981). In fact, we use the diagrams introduced by Veilleux \& Osterbrock (1987): [O III] $] \lambda 25007 / \mathrm{H} \beta$ versus $[\mathrm{N}$ II $] \lambda \lambda 6584 / \mathrm{H} \alpha$, [O III] $\lambda \lambda 5007 / \mathrm{H} \beta$ versus $[\mathrm{O}$ I $] \lambda \lambda 6300 / \mathrm{H} \alpha$,
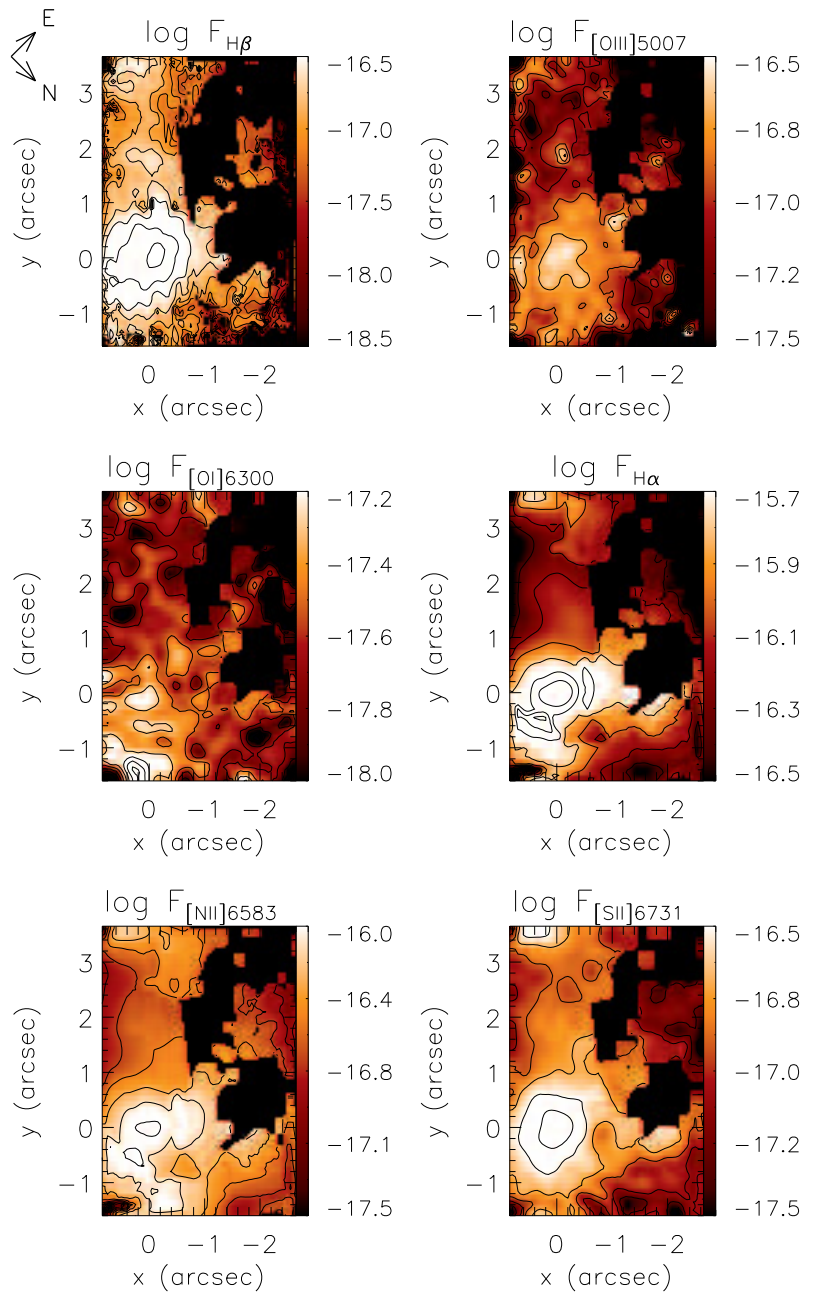

Figure 9. Total flux maps of the $\mathrm{NW}_{a}$. Flux unit are shown in logarithmic scale of $\mathrm{erg} \mathrm{s}^{-1} \mathrm{~cm}^{2}$ fiber $^{-1}$. The black regions demonstrate masked location corresponding to the $\mathrm{NW}_{b}$ and/or lower SNR $(S / N<3)$.

and $[\mathrm{O} \mathrm{III}] \lambda \lambda 5007 / \mathrm{H} \beta$ versus $[\mathrm{S} \mathrm{II}] \lambda \lambda 6716+31 / \mathrm{H} \alpha$, as shown in Fig. 13 (left-hand panels). The point cloud in each diagram represents line ratios computed for individual spaxels, using the fluxes of the blueshifted or redshifted Gaussian components as appropriate. The symbols represent line ratios (see Table 1) derived from spectra extracted from apertures centred on regions (a)-(d) as indicated in Fig. 3. The dashed lines represent the empirical boundaries separating galaxies that have starburst/H II region nuclei (grey points), AGN (blue points), and composite nuclei (green points) derived from analyses of SDSS spectra by Kewley et al. (2001) and Kauffmann et al. (2003). The spaxels occupying each region are colour coded and their distributions over the IFU FOV are shown in the corresponding excitation maps (right-hand panels of Fig. 13).

Also plotted in the BPT diagrams are loci of radiative shock models taken from the library computed with the MAPPINGS III code provided by Allen et al. (2008). These models include a photoionized precursor and assume solar abundances and a preshock density $1.0 \mathrm{~cm}^{-3}$. Sequences in shock velocity ranging from 100 to $1000 \mathrm{~km} \mathrm{~s}^{-1}$ are plotted for three values of the magnetic field strength parameter: $B / \sqrt{n}=10^{-4}, 0.5$ and $1.0 \mu \mathrm{G}$, and a pre-shock density of $1.0 \mathrm{~cm}^{-3}$. 

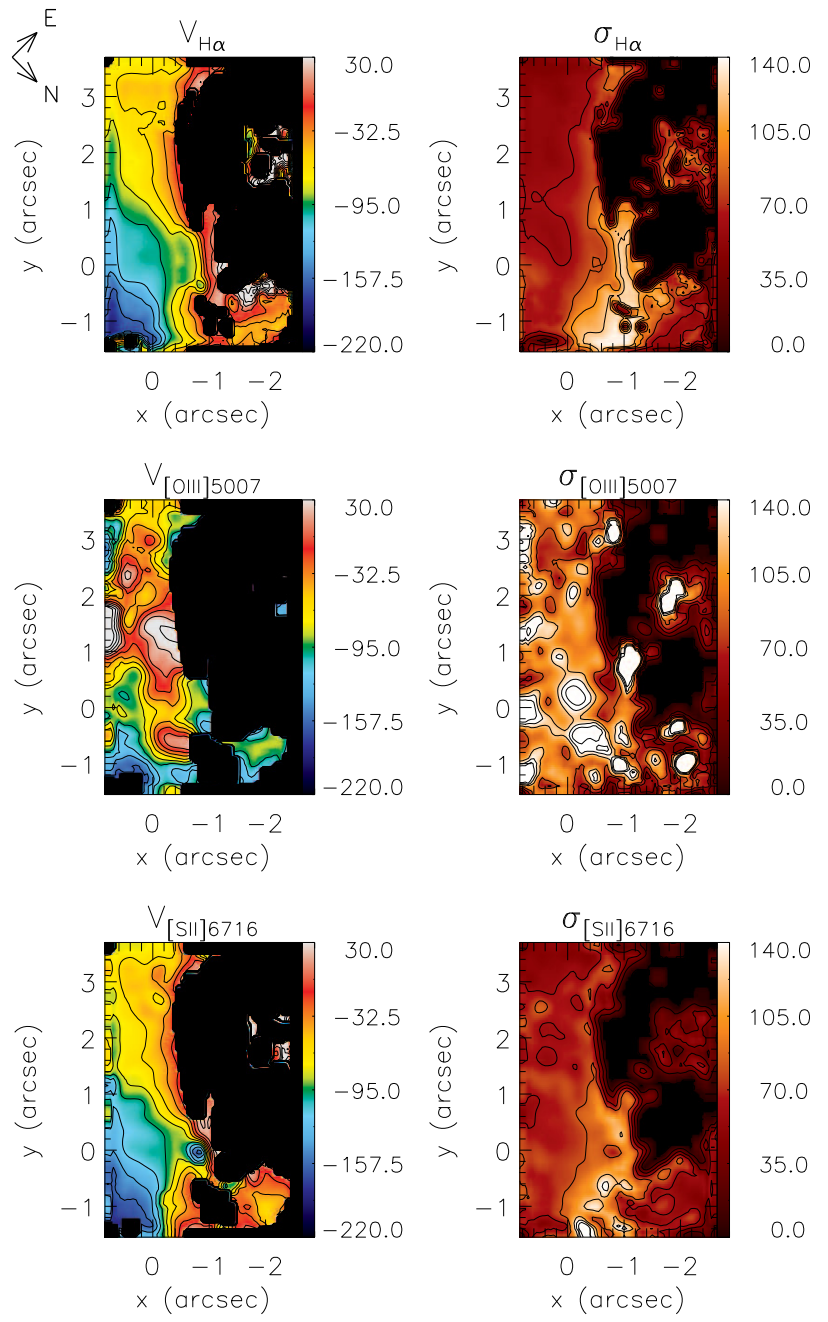

Figure 10. Centroid velocity and velocity dispersion maps for some emission lines of the $\mathrm{NW}_{a}$. Units are in $\mathrm{km} \mathrm{s}^{-1}$. The black regions demonstrate masked location corresponding to the $\mathrm{NW}_{b}$ and/or lower $\operatorname{SNR}(S / N<3)$.

It is apparent that the vast majority of the spaxels fall within the stellar photoionized ' $\mathrm{H}$ II region' zones in the $[\mathrm{O} \mathrm{III}] \lambda \lambda 5007 / \mathrm{H} \beta-$ $[\mathrm{O}$ I] $\lambda \lambda 26300 / \mathrm{H} \alpha$ and $[\mathrm{O} \mathrm{III}] \lambda \lambda 5007 / \mathrm{H} \beta-[\mathrm{S} \mathrm{II}] \lambda \lambda 6716+31 / \mathrm{H} \alpha$ diagrams, or in either the 'H II region' or 'composite' zones in the $[\mathrm{O}$ III $] \lambda \lambda 5007 / \mathrm{H} \beta-[\mathrm{N} \mathrm{II}] \lambda \lambda 6584 / \mathrm{H} \alpha$. However, the distribution also extends into the 'AGN' zone in all three diagrams. Notably, the majority of the spaxels that fall within the latter zone are located in the region close to the NW edge of the FOV that is characterized by high velocity dispersion and relatively strong [O I $] \lambda 6300$ emission (e.g. Figs 11 and 12).

The line ratios derived from the integrated spectra of regions (a)-(c) and (d)-(f) generally cluster quite closely together within the 'H II region' zones (or close to the 'H II region'-'Composite' boundary in the $\mathrm{O}$ III $] \lambda \lambda 5007 / \mathrm{H} \beta-[\mathrm{N}$ II $] \lambda \lambda 6584 / \mathrm{H} \alpha$ diagram). The exception is region (d), which falls close to the 'H II region'-'AGN' or 'composite'-'AGN' boundaries in all three diagrams. This region is also located near the NW edge of the FOV and has a relatively high velocity dispersion.

The distribution of spaxels and selected regions in the BPT diagrams indicates that stellar photoionization is the dominant ionization mechanism over most of the FOV, including the bright blobs, $\mathrm{NW}_{a}$ and $\mathrm{NW}_{b}$, and the more diffuse emission covering
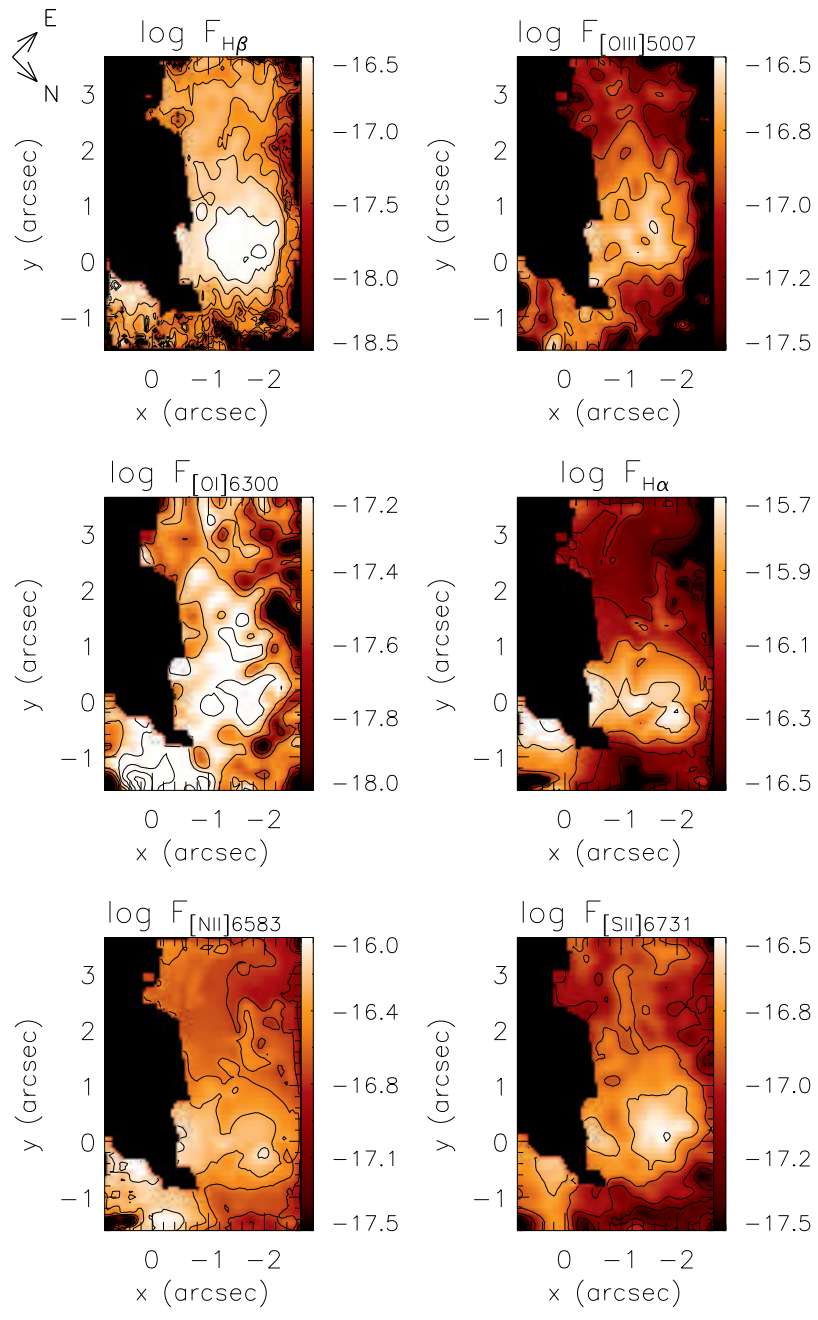

Figure 11. Total flux maps of the blob $b$. Flux unit are shown in logarithmic scale of erg s${ }^{-1} \mathrm{~cm}^{2}$ fiber ${ }^{-1}$. The black regions demonstrate masked location corresponding to the $\mathrm{blob}_{a}$ and/or lower SNR $(S / N<3)$.

most of the rest of the field. However, the region NW of the bright blobs and centred roughly between them along the $x$ axis is characterized by a high velocity dispersion and comparatively large values of the $[\mathrm{N}$ II $] \lambda 6584 / \mathrm{H} \alpha,[\mathrm{O}$ I] $] \lambda 6300 / \mathrm{H} \alpha$, and $[\mathrm{S} \mathrm{II}] \lambda 6716+31 / \mathrm{H} \alpha$ ratios. The line ratios in this region are consistent with weak AGN photoionization (i.e. LINER-like) but given the high velocity dispersion, a more likely explanation is shock ionization. The shock models indeed roughly reproduce the lateral scatter towards the 'AGN' zone in the BPT diagrams and in the $\mathrm{O}$ III $] \lambda \lambda 5007 / \mathrm{H} \beta-[\mathrm{O}$ I $] \lambda \lambda 6300 / \mathrm{H} \alpha$, and $[\mathrm{O}$ III $] \lambda \lambda 5007 / \mathrm{H} \beta-$ $[\mathrm{S} \mathrm{II}] \lambda \lambda 6716+31 / \mathrm{H} \alpha$ diagrams, at least, the region (d) line ratios are consistent with a shock velocity of $\sim 200 \mathrm{~km} \mathrm{~s}^{-1}$. On the other hand, the models do not match the observed line ratios particularly well in the $[\mathrm{O}$ III $] \lambda \lambda 5007 / \mathrm{H} \beta-[\mathrm{N}$ II $] \lambda \lambda 6584 / \mathrm{H} \alpha$ diagram because the models underpredict the $[\mathrm{N}$ II $] \lambda \lambda 6584 / \mathrm{H} \alpha$ ratio for a given shock velocity.

Nevertheless, it seems reasonable to conclude that the line emission in region (d) and in the blue-shaded regions NW of the bright blobs in Fig. 13 predominantly arises due to shock ionization. As can be seen in the excitation map corresponding to the $[\mathrm{O}$ III $] \lambda \lambda 5007 / \mathrm{H} \beta-[\mathrm{N}$ II $] \lambda \lambda 6584 / \mathrm{H} \alpha$ diagram, the 'shockionized' region is surrounded by green-shaded 'composite' region 

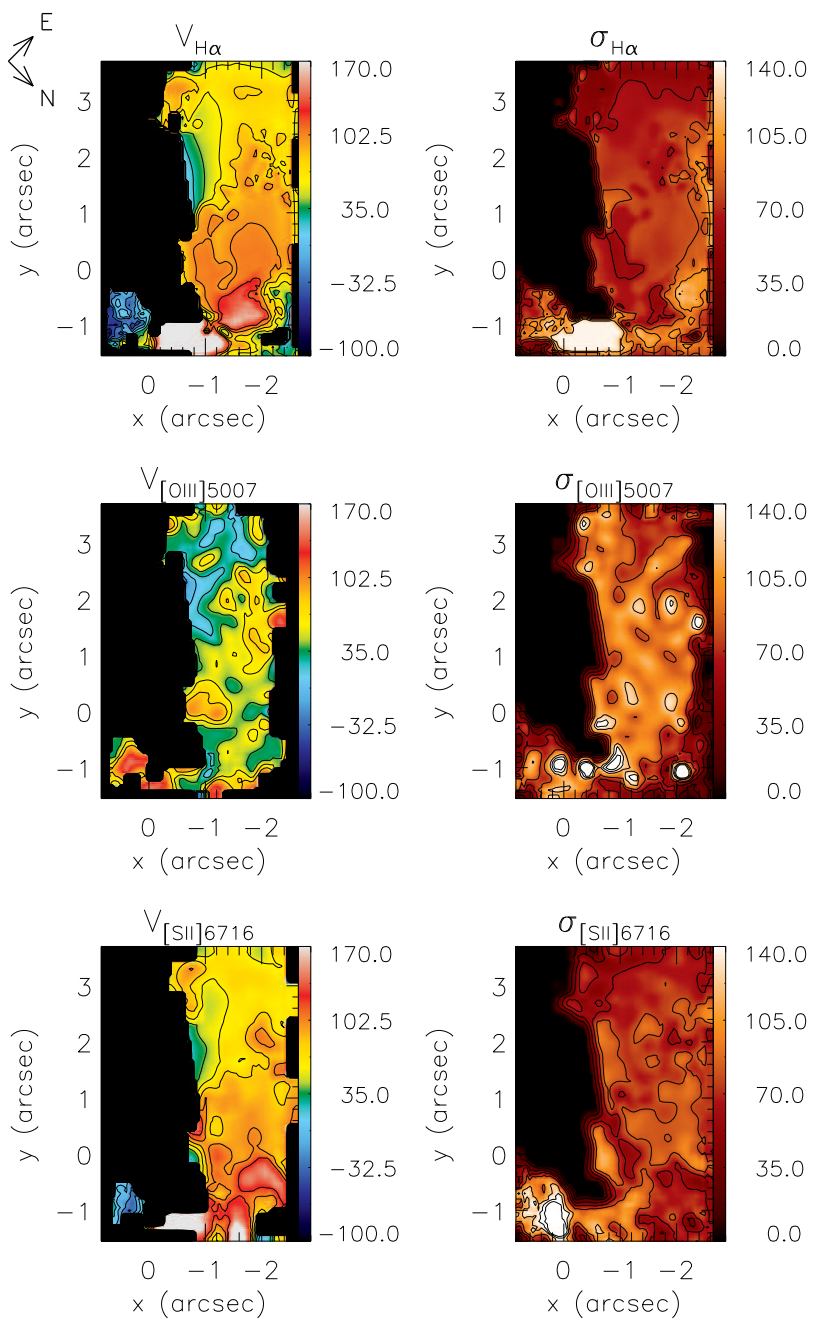

Figure 12. Examples of the centroid velocity and velocity dispersion maps for some emission lines of the $\mathrm{NW}_{b}$. Units are in $\mathrm{km} \mathrm{s}^{-1}$. The black regions demonstrate masked location corresponding to the $\mathrm{NW}_{b}$ and/or lower SNR $(S / N<3)$.

spaxels. This may indicate a transition zone where both shocks and $\mathrm{H}$ II regions contribute to the spectrum.

\section{4 $\mathrm{H} \alpha$ photometry and star formation rates}

As star formation appears to dominate the line emission from most of the IFU FOV, including the two brightest blobs (Section 3.3), $\mathrm{H} \alpha$ fluxes measured from the continuum subtracted FR656N ramp filter image were used to estimate star formation rates (SFRs). Flux measurements were made for the entire IRAS17526 + 3253 system and also within smaller apertures centred on each of the two merging galaxies, the IFU field, and blobs $\mathrm{NW}_{a}$ and $\mathrm{NW}_{b}$ within the IFU field. The aperture sizes used for the photometric measurements are listed in Table 2. In order to determine the intrinsic $\mathrm{H} \alpha$ fluxes from these measurements, it is necessary to correct for both dust extinction and the contribution of the $[\mathrm{N} I I] \lambda \lambda 6548,6584$ lines, which fall within the filter passband. Approximate corrections were derived using line intensity ratios obtained from the IFU data. As there are large changes in the Balmer decrement over the IFU FOV, we derived two values for the $\mathrm{H} \alpha$ extinction, one using the $\mathrm{H} \alpha$ and $\mathrm{H} \beta$ line fluxes integrated over the whole FOV, and the other using average of the line fluxes measured in 1 arcsec apertures centred on blobs $\mathrm{NW}_{a}$ and $\mathrm{NW}_{b}$. For the IFU/FOV, we find $I_{\mathrm{H} \alpha} / I_{\mathrm{H} \beta} \approx$ 8.0, yielding $A_{\mathrm{H} \alpha} \approx 2.7$, using the standard Milky Way extinction curve (Cardelli, Clayton \& Mathis $1989 ; R_{V}=3.1$ ) and assuming the Case B recombination value, $I_{\mathrm{H} \alpha} / I_{\mathrm{H} \beta}=2.87$, for the intrinsic Balmer decrement (Osterbrock \& Ferland 2006). For the blobs, the average Balmer decrement is $I_{\mathrm{H} \alpha} / I_{\mathrm{H} \beta} \approx 4.7$, implying a much lower extinction, $A_{\mathrm{H} \alpha} \approx 1.3$.

Inspection of the FR656N image suggests that it is reasonable to suppose that the extinction affecting the IFU field is fairly representative of the emission line regions elsewhere in the system. Therefore, assuming that the two values of $\mathrm{A}_{\mathrm{H} \alpha}$ derived from the IFU data represent upper and lower limits on the amount of extinction affecting a given region, we have used both values to calculate extinction corrections for each component, with the exception of the blobs themselves for which only the corresponding local value of $\mathrm{A}_{\mathrm{H} \alpha}$ was used.

After applying extinction corrections, values of the $[\mathrm{N}$ II] $]$ 6584/H $\alpha$ ratio measured from the IFU field were used to estimate and remove the contribution of the $[\mathrm{N} \mathrm{II}] \lambda \lambda 6548,6584$ lines to the fluxes measured from the HST images. Again, separate values of this ratio were derived, corresponding to the the whole IFU/FOV, and the average of the two blobs. Assuming $I_{[\mathrm{N} \mathrm{II}] \lambda 6584} / I_{[\mathrm{N} \mathrm{II}] \lambda 6548}=2.9$, we estimate $\mathrm{H} \alpha$ contributions to the extinction-corrected fluxes of 65 percent and 73 percent, respectively. The measured $H \alpha+[\mathrm{NII}]$ fluxes, the reddeningcorrected $\mathrm{H} \alpha$ fluxes and the $\mathrm{H} \alpha$ luminosities are listed for each region for both the 'high' and 'low' extinction cases in Table 2.

The corresponding SFRs are also listed in Table Table 3 and were estimated from the $\mathrm{H} \alpha$ luminosity using the relationship given by Calzetti et al. (2007): $\operatorname{SFR}_{\mathrm{H} \alpha}\left(\mathrm{M}_{\odot} \mathrm{yr}^{-1}\right)=5.3 \times 10^{-42} \mathrm{~L}_{\mathrm{H} \alpha}$. The measured $\mathrm{H} \alpha$ luminosities imply that the unobscured SFR for the entire IRAS17526 + 3253 system is $\sim 10-30 \mathrm{M}_{\odot} \mathrm{yr}^{-1}$, with the central regions of the interacting galaxies contributing $\sim 2.6-7.9$ and $\sim 1.5-4.5 \mathrm{M}_{\odot} \mathrm{yr}^{-1}$ for the NW and east components, respectively, and with most of the star formation in the latter being associated with the bright $\mathrm{H}$ II regions within the IFU/FOV.

\section{DISCUSSION}

\subsection{Large-scale morphology and merger stage}

Our HST/ACS observations confirm earlier suggestions (Andreasian \& Alloin 1994; Wagner 2013) that IRAS17526 + 3253 is a late-stage major merger. Although the NW nucleus appears larger and brighter in the F814W image and has a brighter radio source, the $\mathrm{K}_{s}$ magnitudes derived from the 2MASS image suggest that the two galaxies have similar bulges, with a mass ratio $\sim 1$. Both galaxies appear to be quite highly inclined, and the elongated nature of the system as a whole suggests that it is viewed close to the orbital plane of the interaction. The two galaxy nuclei are separated by a projected distance of $\sim 9 \mathrm{kpc}$, and appear to be embedded within a complex, disturbed structure with multiple dust lanes, large-scale tidal tails or plumes and widely distributed clumps of star formation. A comparison with merger simulations (e.g. Lotz et al. 2008; Hung et al. 2016) suggests that the morphological properties of the system are consistent with a mid to advanced merger stage, probably between the second pericenter passage and final coalescence.

Both nuclei host compact, but resolved radio sources at $1.49 \mathrm{GHz}$; which have properties consistent with radio emission from starburst galaxies (Baan \& Klöckner 2006). An optical spectrum obtained 

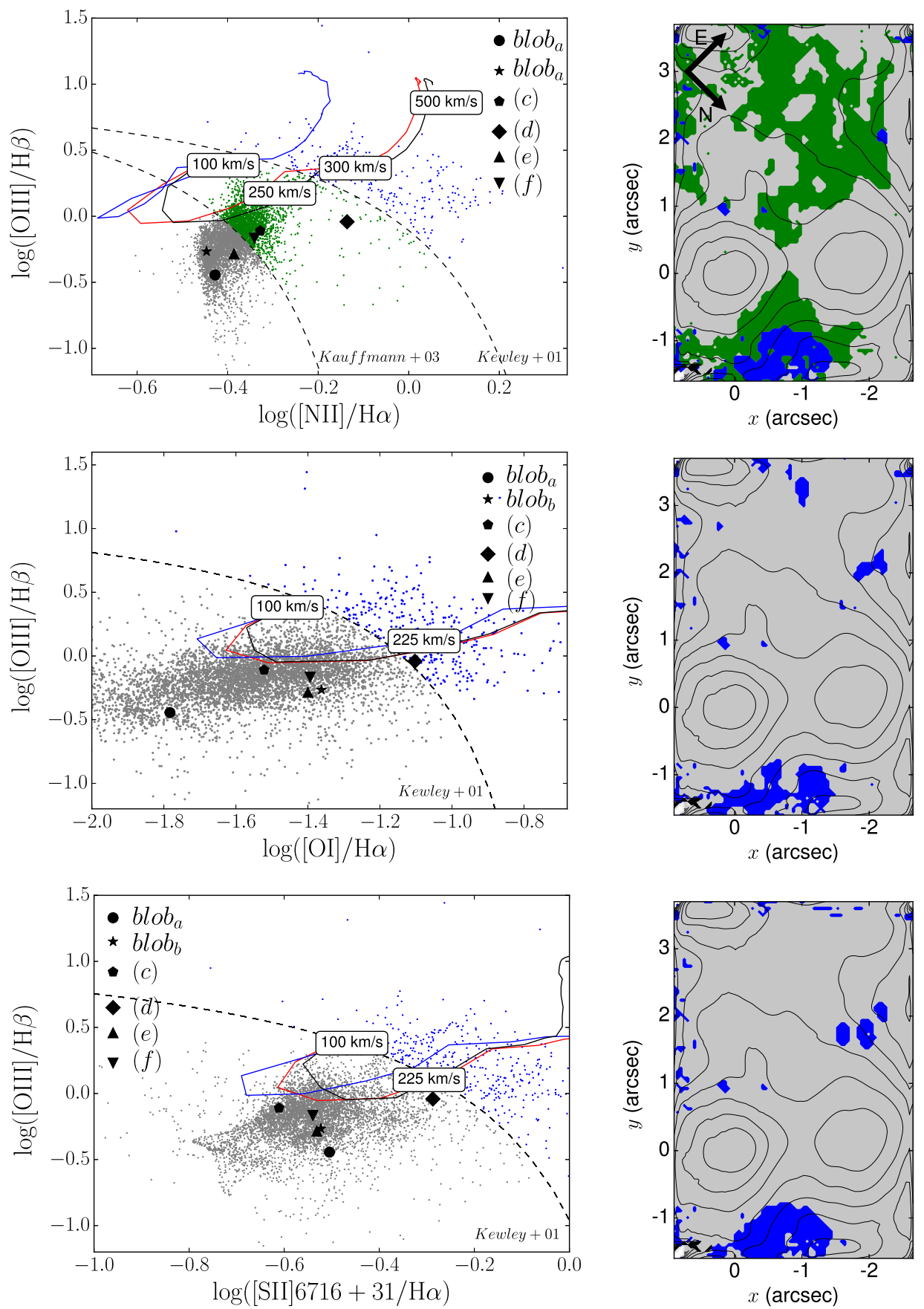

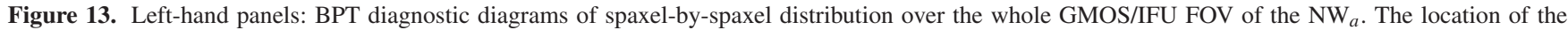
dividing lines are taken from Kewley et al. (2001). Green: composites. Grey: H II regions and blue are Seyferts. Then red solid lines represent shock + precursor models with solar metallicity, shock velocities in the range of $100-1000 \mathrm{~km} \mathrm{~s}^{-1}$ in steps of $25 \mathrm{~km} \mathrm{~s}^{-1}$, magnetic parameters $\mathrm{B} / \sqrt{n}$ of $0.5 \mu \mathrm{G}$, and pre-shock density of $1.0 \mathrm{~cm}^{-3}$. The blue and black solid lines are the same to magnetic parameters $\mathrm{B} / \sqrt{n}$ of $10^{-4} \mu \mathrm{G}$ and $1.0 \mu \mathrm{G}$, respectively. Right-hand panels: The blue, green and grey points are spatially represented by the filled regions overplotted on $\mathrm{H} \alpha$ map (right-hand panel).

by Baan et al. (1998), is also consistent with starburst activity, but this classification probably relates only to the NW nucleus (private communication).

Our IFU observation was designed to sample the brightest emission line region in the NW galaxy. As discussed in (3), this region has a complex structure with at least two kinematically distinct components, but over most of the FOV, the emission line ratios are consistent with photoionization in star formation regions. However, the radio source associated with this galaxy is centred within the prominent dust lane that borders the northern corner of the IFU field. Thus, assuming that the radio source core corresponds to the nucleus of the galaxy, it is evidently embedded in the dust lane and heavily obscured at optical wavelengths. It is likely therefore that the IFU field does not include the nucleus itself, but samples the surrounding star formation regions. 
Table 1. Line Ratios derived from GMOS/IFU data.

\begin{tabular}{lcccc}
\hline & $\log \left(\frac{\mathrm{H} \alpha}{[\mathrm{NII}]}\right)$ & $\log \left(\frac{[\mathrm{O} \mathrm{III}]}{\mathrm{H} \beta}\right)$ & $\log \left(\frac{[\mathrm{O} \mathrm{O}]}{\mathrm{H} \alpha}\right)$ & $\log \left(\frac{[\mathrm{S} \mathrm{II}]}{\mathrm{H} \alpha}\right)$ \\
\hline Blob $_{a}$ & -0.42 & -0.44 & -1.78 & -0.50 \\
Blob $_{b}$ & -0.45 & -0.26 & -1.36 & -0.52 \\
Region (c) & -0.33 & -0.11 & -1.52 & -0.61 \\
Region (d) & -0.14 & -0.04 & -1.10 & -0.29 \\
Region (e) & -0.39 & -0.28 & -1.40 & -0.53 \\
Region (f) & -0.34 & -0.17 & -1.39 & -0.54 \\
\hline
\end{tabular}

Table 2. $\mathrm{H} \alpha$ luminosities and SFRs measured from HST images.

\begin{tabular}{|c|c|c|c|c|}
\hline $\begin{array}{l}\text { Flux } \\
\text { Aperture radius }\end{array}$ & $\begin{array}{c}\text { Northwestern } \\
5 .\end{array}$ & $\begin{array}{c}\text { East } \\
5 !\end{array}$ & $\begin{array}{l}\text { System } \\
27 " .\end{array}$ & $\begin{array}{l}\text { IFU/FOV } \\
5^{\prime \prime} .1 \times 3 . ! 4\end{array}$ \\
\hline \multicolumn{5}{|c|}{ Reddening and [N II] correction derived from IFU/FOV } \\
\hline $\begin{array}{l}\left.F_{\mathrm{H} \alpha+[\mathrm{NII}}\right] \\
\left(\mathrm{erg} \mathrm{s}^{-1} \mathrm{~cm}^{-2}\right)\end{array}$ & $1.4 \mathrm{e}-13$ & $8.3 \mathrm{e}-14$ & $6.0 \mathrm{e}-13$ & $1.7 \mathrm{e}-14$ \\
\hline$F_{\mathrm{H} \alpha, \text { corr }}$ & $1.1 \mathrm{e}-12$ & $6.1 \mathrm{e}-13$ & $4.5 \mathrm{e}-12$ & $4.0 \mathrm{e}-13$ \\
\hline $\mathrm{L}_{\mathrm{H} \alpha}\left(\mathrm{erg} \mathrm{s}^{-1}\right)$ & $1.5 \mathrm{e}+42$ & $8.5 e+41$ & $6.2 \mathrm{e}+42$ & $5.6 \mathrm{e}+41$ \\
\hline $\mathrm{SFR}\left(\mathrm{M}_{\odot} \mathrm{yr}^{-1}\right)$ & 7.9 & 4.5 & 33.1 & 2.98 \\
\hline \multicolumn{5}{|c|}{$\begin{array}{l}\text { Reddening and [N II] correction derived using } \\
\text { an average value from IFU } \mathrm{NW}_{a} \text { and } \mathrm{NW}_{b}\end{array}$} \\
\hline $\begin{array}{l}\left.F_{\mathrm{H} \alpha+[\mathrm{N}} \mathrm{II}\right] \\
\left(\mathrm{erg} \mathrm{s}^{-1} \mathrm{~cm}^{-2}\right)\end{array}$ & $1.4 \mathrm{e}-13$ & $8.3 e-14$ & $6.0 \mathrm{e}-13$ & $1.7 \mathrm{e}-14$ \\
\hline$F_{\mathrm{H} \alpha, \text { corr }}$ & $3.5 e-13$ & $2.0 \mathrm{e}-13$ & $1.5 \mathrm{e}-12$ & $1.3 \mathrm{e}-13$ \\
\hline$L_{\mathrm{H} \alpha}\left(\mathrm{erg} \mathrm{s}^{-1}\right)$ & $4.9 e+41$ & $2.8 \mathrm{e}+41$ & $2.0 \mathrm{e}+42$ & $1.8 \mathrm{e}+41$ \\
\hline $\mathrm{SFR}\left(\mathrm{M}_{\odot} \mathrm{yr}^{-1}\right)$ & 2.58 & 1.48 & 10.8 & 0.97 \\
\hline
\end{tabular}

Table 3. SFR of IRAS $17526+3253$.

\begin{tabular}{lcccccc}
\hline Flux & Northwestern & East & System & $\mathrm{NW}_{a}$ & $\mathrm{NW}_{b}$ & $\mathrm{IFU} / \mathrm{FOV}$ \\
\hline $\mathrm{H} \alpha(H S T)$ & 7.9 & 4.5 & 33.1 & 0.3 & 0.2 & 3.0 \\
$\mathrm{H} \alpha(\mathrm{IFU})$ & - & - & - & 4.1 & 2.8 & 12.0 \\
$\mathrm{~L}_{\mathrm{FIR}}{ }^{a}$ & - & - & 26.8 & - & - & - \\
$\mathrm{L}_{1.4 \mathrm{GHz}}{ }^{b}$ & 13.1 & 6.0 & - & - & - & - \\
$\mathrm{L}_{1.4 \mathrm{GHz}}{ }^{a}$ & - & - & 13.7 & - & - & - \\
\hline
\end{tabular}

${ }^{a}$ IIFSCz, Wang \& Rowan-Robinson (2009).

${ }^{b}$ Baan \& Klöckner (2006).

The Imperial IRAS-FSC Redshift Catalogue (IIFSCz Wang \& Rowan-Robinson 2009) gives an FIR luminosity of $L_{\mathrm{FIR}}=$ $10^{11.19} \mathrm{~L}_{\odot}$ for the IRAS17526 + 3253 system, implying a global SFR $S F R_{\mathrm{FIR}} \sim 27 \mathrm{M}_{\odot} \mathrm{yr}^{-1}$. This is approximately consistent with the total of the obscured and unobscured SFRs estimated from the 1.4 GHz radio sources (Baan \& Klöckner 2006) and the continuumsubtracted $\mathrm{H} \alpha+[\mathrm{N}$ II] image, respectively (Section 3.4). The FIR luminosity and global SFR are both somewhat higher than the median values $\left(\sim 10^{11} \mathrm{~L}_{\odot}\right.$ and $\sim 15 \mathrm{M}_{\odot} \mathrm{yr}^{-} 1$, respectively) for the sample of $\sim 3000$ local mergers studied by Carpineti et al. (2015), but are very close to the peaks of the respective distributions. Evidently, IRAS17526 + 3253 is a fairly typical example of a spiral-spiral merger in the local universe, in so far as its SFR is concerned.

\subsection{Ionized gas excitation and kinematics}

As noted above, the IFU field samples a bright emission line region within the northern galaxy, bordering the compact radio source that we presume locates the obscured nucleus. As discussed in Section 3.2, the emission line region is divided into two main components by a discontinuity in the velocity field of magnitude $\sim 200 \mathrm{~km} \mathrm{~s}^{-1}$, one of which (on the SW side) is blueshifted and the other (NE side) redshifted, relative to the mean velocity. These components are also present in the velocity channel maps and are also clearly visible in the PCA tomograms as eigenvectors 1 and 2, which together account for 95 per cent of the variance. In addition, the increased velocity dispersion and line splitting along the boundary defined by the velocity field discontinuity are also consistent with physically distinct components at different velocity shifts, with one slightly overlapping the other in projection. Morphologically, each kinematic component is dominated by a bright blob ( $\mathrm{NW}_{a}$ and $\mathrm{NW}_{b}$, respectively), appearing both in continuum and in line emission, which are separated by $\sim 850 \mathrm{pc}$, and surrounded by more diffuse ionized gas.

The emission line ratios indicate that star formation is the main source of the line emission in both kinematic components, including the two bright blobs. However, the fainter line emission bordering the blobs to the north-west is characterized by a higher velocity dispersion and line ratios consistent with shock ionization for shock velocities $\sim 200 \mathrm{~km} \mathrm{~s}^{-1}$. This shock ionized region is situated between the bright blobs and both the nuclear radio source and the prominent dust lane, which crosses the northern corner of the IFU field.

The origin of the two kinematic components is unclear. However, it seems plausible that one is associated with the disrupted disc of the NW galaxy, while the other is tidal debris, seen in projection and partially overlapping the disc. It is possible that this 'debris' is a tidal tail originating in the SE galaxy since the long-slit spectrum obtained by Andreasian \& Alloin (1994) suggests that this galaxy is redshifted by $\sim 300 \mathrm{~km} \mathrm{~s}^{-1}$ relative to its NW counterpart. The HST F814W image suggests that the prominent dust lane crossing the core of NW galaxy may also be associated with the tidal tail. In this scenario, the shocked gas may be the result of an interaction between the tidal tail and part of the NW galaxy's disc.

\subsection{Maser sources}

As noted in Section 1, the identification of an $\mathrm{OH}$ maser source in IRAS17526 + 3253 rests on the feature reported by Martin (1989), which has a velocity $\approx 7500 \mathrm{~km} \mathrm{~s}^{-1}$. In comparison, the H I $21 \mathrm{~cm}$ emission observed by the same author peaks at $\approx 7800 \mathrm{~km} \mathrm{~s}^{-1}$, approximately the same velocity as the $\mathrm{H}_{2} \mathrm{O}$ maser lines (Wagner 2013). In addition, $\mathrm{CO}$ emission has been detected both at $\approx 7500$ and $\approx 7800 \mathrm{~km} \mathrm{~s}^{-1}$ (Baan et al. 2008). There are evidently two velocity systems present in the IRAS17526 + 3253 system, which are most probably associated with the two galaxies revealed by the HST image. The $\mathrm{OH}$ maser emission and the blueshifted component of the $\mathrm{CO}(2-1)$ line have velocities comparable with that of the blueshifted component of ionized gas, which we have identified with the NW galaxy. As discussed above (Section 4.2), the SE galaxy appears to be redshfted by $\sim 300 \mathrm{~km} \mathrm{~s}^{-1}$ with respect to the latter. Based on the kinematics therefore we can conclude that the $\mathrm{OH}$ maser source is located in the NW galaxy, whereas the $\mathrm{H}_{2} \mathrm{O}$ masers are associated with the SE galaxy.

\subsection{Comparison with previously studied OHMG}

IRAS17526 + 3253 is the fourth OHMG that we have studied in detail to date. These systems exhibit a range of morphological and gas-excitation characteristics. Multiwavelength imaging and spectral energy distribution analysis of IRAS16399-0937 (Sales et al. 2015) revealed that it is a mid to advanced merger with two nuclei embedded in a tidally distorted envelope, with a total SFR $\sim 20 \mathrm{M}_{\odot} \mathrm{yr}^{-1}$. The nuclei are separated by $\sim 3.4 \mathrm{kpc}$ and the 
NW hosts a dust-embedded AGN of luminosity $L_{\text {bol }} \sim 10^{44} \mathrm{erg} \mathrm{s}^{-1}$. IRASF23199 + 0123 was studied using HST and VLA images as well as Gemini IFU spectroscopy (Hekatelyne et al. 2018a). This is an interacting pair, connected by a tidal tail, whose two galaxies are separated by $24 \mathrm{kpc}$. The detection of a broad $\mathrm{H} \alpha$ emission line revealed that the eastern member of the pair hosts a Seyfert 1 nucleus. In this case, we were also able to obtain VLA maps the $\mathrm{OH}$ maser emission, showing that the eastern galaxy also hosts two masing sources, which appear to be closely associated with the AGN. The $\mathrm{OH}$ masers are located in the vicinity of a region of enhanced velocity dispersion and higher $[\mathrm{NII}] / \mathrm{H} \alpha$ ratios, suggesting that they are associated with shocks driven by AGN outflows. In contrast, IRAS03056 + 2034 is a barred spiral with morphological irregularities suggesting interactions and a circumnuclear ring (radius $\sim 0.5 \mathrm{kpc}$ ) of star-forming regions, with an SFR $\sim 5 \mathrm{M}_{\odot} \mathrm{yr}^{-1}$ (Hekatelyne et al. 2018b). As in IRAS17526 + 3253, there is no clear evidence that the galaxy hosts an AGN.

These four systems are too small a sample upon which to base any firm conclusions. However, it is perhaps notable that $\mathrm{OH}$ masers are present in mid to advanced major mergers (IRAS16399-0937, IRAS17526+3253) as well as in a galaxy that may be in an early stage of a possible merger (IRASF23199+0123) and one that is not undergoing any noticeable interaction with a similarly sized companion (IRAS03056+2034). Two of these systems harbour moderately luminous embedded AGN in at least one of their components (IRAS16399-0937 and IRASF23199 + 0123), but the other two appear to be dominated by star formation. In the one case for which we have so far been able to establish the location of the $\mathrm{OH}$ maser sources, they are associated with the AGN, perhaps arising in shocks driven by the latter.

IRAS17526 + 3253 is one of only a handful of galaxies known to host both luminous $\mathrm{OH}$ and $\mathrm{H}_{2} \mathrm{O}$ masers (Wiggins, Migenes \& Smidt 2016). Of these, only two are dual megamaser hosts, Arp 299, (Tarchi et al. 2011) and II Zw 96, (Wagner 2013; Wiggins et al. 2016; a possible third candidate, UGC5101, has a dubious $\mathrm{OH}$ detection; Wiggins et al. 2016). The apparent dearth of systems hosting megamasers of both species may be due to the different physical conditions required for maser emission in each case, with $\mathrm{OH}$ masers typically occurring in (U)LIRG systems, whereas $\mathrm{H}_{2} \mathrm{O}$ masers are usually associated with AGN (Lo 2005). The two dual megamaser hosts are both merging systems with spatially distinct nuclei and both are spectroscopically classified as $\mathrm{H}$ II galaxies. Although IRAS $7526+3253$ does not quite qualify as an $\mathrm{OH}$ megamaser host by the criteria adopted by Wiggins et al. 2016 $\left(L_{\mathrm{OH}}>10 \mathrm{~L}_{\odot}\right)$, it's $\mathrm{OH}$ maser luminosity is only slightly less than that of Arp 299. On the other hand, IRAS $7526+3253$ has an $\mathrm{H}_{2} \mathrm{O}$ maser luminosity comparable with that of the other two systems. In several respects therefore IRAS $17526+3253$ is similar to the two known dual megamaser hosts. In Arp 299, the $\mathrm{H}_{2} \mathrm{O}$ maser emission was detected in the nucleus that also hosts the $\mathrm{OH}$ maser source (Tarchi et al. 2011). The II Zw 96 system appears to be a complex advanced merger in which the $\mathrm{OH}$ maser source is associated with a tidally stripped and heavily reddened nucleus that may contain an embedded AGN (Migenes et al. 2011), but it is not clear if the $\mathrm{H}_{2} \mathrm{O}$ maser comes from the same region. In IRAS $7526+3253$, the evidence suggests that the $\mathrm{OH}$ and $\mathrm{H}_{2} \mathrm{O}$ masers are associated with different nuclei (NW and SE, respectively). There is no evidence supporting the presence an AGN in either nucleus, although this possibility cannot be ruled based on the existing data, and we currently lack a spectroscopic classification for the SE nucleus. Thus, further observations of IRAS $17526+3253$, in particular, to determine if either nucleus hosts an AGN may shed light on any connections between $\mathrm{OH}$ and $\mathrm{H}_{2} \mathrm{O}$ masers and galaxy merger stage.

\section{SUMMARY AND CONCLUSIONS}

We have presented a multiwavelength study of the luminous infrared galaxy IRAS17526 + 3253, using Gemini GMOS/IFU, HST, 2MASS, and VLA data. Previous radio studies have classified the two major components of the IRAS17526 + 3253 system as starburst galaxies. This system has also been reported to host $\mathrm{OH}$ megamaser emission, although strong radio frequency interference frustrated a more recent attempt to confirm the detection.

The data set analysed here combines new emission line ( $\mathrm{H} \alpha+[\mathrm{N}$ II) and broad-band (FR914M, F814W) imaging obtained with the HST ACS Wide Field Camera with archival VLA radio imaging. We also present a 2D analysis of the gas excitation and kinematics of a bright emission line region in the NW galaxy. The main results from this analysis are summarized next:

(i) The HST/ACS, 2MASS Ks band, and $1.49 \mathrm{GHz}$ VLA images clearly reveal a mid-stage major merger with two main galaxy nuclei separated by a projected distance of $\sim 8.5 \mathrm{kpc}$, embedded in an elongated irregular envelope. The NW galaxy is quite highly inclined, with the second component appearing to be merging with its companion and probably between the second pericenter passage and final coalescence. The morphological elongation of the system suggests that we are observing it from a viewpoint quite close to the orbital plane. The two nuclei are clearly distinguished in the NIR (2MASS $K s$ band) and each is associated with a compact (but resolved) $1.49 \mathrm{GHz}$ radio source, previously attributed to star formation. The HST/ACS F814W image shows that the two galaxies are embedded in a large tidally distorted envelope with a complex structure including numerous dust lanes and bright knots.

(ii) The HST $\mathrm{H} \alpha+[\mathrm{N}$ II emission line image shows at least 27 compact regions of ongoing star formation across the envelope over scales of several tens of kpc. IRAS17526 +3253 has previously been classified, based on an optical spectrum as a star-forming galaxy.

(iii) Our Gemini/IFU observation was designed to sample the brightest emission line region in the northern galaxy, which lies just outside the galaxy nucleus. Assuming that the radio core corresponds to the position of the latter, it is evidently obscured by a prominent dust lane that borders the northern corner of the Gemini/IFU FOV. Two distinct kinematic components, divided by a discontinuity in the velocity field of magnitude $\sim 200 \mathrm{~km} \mathrm{~s}^{-1}$, are present in the IFU field. One component (on the SW side) is blueshifted and the other (NE side) redshifted, relative to the mean velocity. These components are also present in the velocity channel maps and are also clearly visible in the PCA tomograms. Each kinematic component is dominated by a bright blob $\left(\mathrm{NW}_{a}\right.$ and $\mathrm{NW}_{b}$, respectively), appearing both in optical continuum and in line emission, which are separated by $\sim 850 \mathrm{pc}$, and surrounded by more diffuse ionized gas.

(iv) The emission line ratio diagnostic diagrams indicate that star formation is the main source of the line emission in both kinematic components, including the two bright blobs. However, the fainter line emission bordering the blobs to the NW is characterized by a higher velocity dispersion and line ratios consistent with shock ionization for shock velocities $\sim 200 \mathrm{~km} \mathrm{~s}^{-1}$. This shock ionized region is situated between the bright blobs and both the nuclear 
radio source and the prominent dust lane crossing the northern corner of the field.

(v) We suggest that the two kinematic components represent, on the SW side of the IFU field, the disrupted disc of the NW galaxy, and on the NE side, tidal debris, seen in projection and partially overlapping the disc. This material may be part of a tidal tail from the SE galaxy. The shocked gas may be the result of an interaction between the tidal tail and part of the NW galaxy's disc.

(vi) The measured $\mathrm{H} \alpha$ luminosities imply that the unobscured SFR of the whole IRAS17526 + 3253 system is $\sim 10-30 \mathrm{M}_{\odot} \mathrm{yr}^{-1}$, with the central regions of the interacting galaxies contributing $\sim 2.6-7.9$ and $\sim 1.5-4.5 \mathrm{M}_{\odot} \mathrm{yr}^{-1}$ for the $\mathrm{NW}$ and east components, respectively, and with most of the star formation in the NW being associated with the bright $\mathrm{H}$ II regions within the IFU/FOV.

(vii) IRAS17526 +3253 is one of only a few systems known to host luminous $\mathrm{OH}$ and $\mathrm{H}_{2} \mathrm{O}$ masers. The velocities of the $\mathrm{OH}$ and $\mathrm{H}_{2} \mathrm{O}$ masers suggest that they are associated with the NW and $\mathrm{SE}$ galaxies, respectively.

\section{ACKNOWLEDGEMENTS}

Support for program HST-SNAP 11604 was provided by NASA through a grant from the Space Telescope Science Institute, which is operated by the Association of Universities for Research in Astronomy, Inc., under NASA contract NAS 5-26555. This material is based upon work partly supported by the National Aeronautics and Space Administration under Grant No. NNX11AI03G issued through the Science Mission Directorate. DAS gratefully acknowledges partial financial support received from 'Fundação de Amparo à Pesquisa do Estado do Rio Grande do Sul/Coordenação de Aperfeiçoamento de Pessoal de Nível Superior (FAPERGS/CAPES) n.05/2013 and Conselho Nacional de Desenvolvimento Científico e Tecnológico (CNPq) Universal 01/2016. AR and DAS acknowledge the Sociedade Brasileira de Física (SBF) and the American Physical Society (APS) for financial support received from the Brazil-U.S. Exchange Program. The work of SB and CO was supported by NSERC (Natural Science and Engineering Research Council of Canada). Based on observations obtained at the Gemini Observatory (include additional acknowledgement here, see section 1.2), which is operated by the Association of Universities for Research in Astronomy, Inc., under a cooperative agreement with the NSF on behalf of the Gemini partnership: the National Science Foundation (United States), the National Research Council (Canada), CONICYT (Chile), Ministerio de Ciencia, Tecnología e Innovaci n Productiva (Argentina), and Ministério da Ciência, Tecnologia e Inovacação (Brazil). This research has used the NASA/IPAC Extragalactic Database (NED), which is operated by the Jet Propulsion Laboratory, California Institute of Technology, under contract with the National Aeronautics and Space Administration.

\section{REFERENCES}

Allen M. G., Groves B. A., Dopita M. A., Sutherland R. S., Kewley L. J., 2008, ApJS, 178, 20

Allington-Smith J. et al., 2002, Publ. Astron. Soc. Pac., 114, 892

Andreasian N., Alloin D., 1994, A\&AS, 107, 23

Angulo R. E., Springel V., White S. D. M., Jenkins A., Baugh C. M., Frenk C. S., 2012, MNRAS, 426, 2046

Baan W. A., Salzer J. J., LeWinter R. D., 1998, ApJ, 509, 633

Baan W. A., Klöckner H.-R., 2006, A\&A, 449, 559

Baan W. A., Henkel C., Loenen A. F., Baudry A., Wiklind T., 2008, A\&A, 477,747
Baldwin J. A., Phillips M. M., Terlevich R., 1981, PASP, 93, 5

Banerji M., Chapman S. C., Smail I., Alaghband-Zadeh S., Swinbank A. M., Dunlop J. S., Ivison R. J., Blain A. W., 2011, MNRAS, 418, 1071

Barnes J. E., Hernquist L., 1992, ARA\&A, 30, 705

Bell E. F., McIntosh D. H., Katz N., Weinberg M. D., 2003, ApJS, 149, 289

Blumenthal G. R., Faber S. M., Primack J. R., Rees M. J., 1984, Nature, 311,517

Boylan-Kolchin M., Springel V., White S. D. M., Jenkins A., Lemson G., 2009, MNRAS, 398, 1150

Calzetti D. et al., 2007, ApJ, 666, 870

Caputi K. I. et al., 2007, ApJ, 660, 97

Cardelli J. A., Clayton G. C., Mathis J. S., 1989, ApJ, 345, 245

Carpineti A., Kaviraj S., Hyde A. K., Clements D. L., Schawinski K., Darg D., Lintott C. J., 2015, A\&A, 577, A119

Cicone C. et al., 2014 , A\&A, 562, A21

Condon J. J., Broderick J. J., 1991, AJ, 102, 1663

Condon J. J., Condon M. A., Broderick J. J., Davis M. M., 1983, AJ, 88, 20 Conselice C. J., 2014, ARA\&A, 52, 291

de Vaucouleurs G., de Vaucouleurs A., Corwin H. G., Jr., Buta R. J., Paturel G., Fouqué P., 1991, Third Reference Catalogue of Bright Galaxies. Volume I: Explanations and references. Volume II: Data for galaxies between $0^{h}$ and $12^{h}$. Volume III: Data for galaxies between $12^{h}$ and $24^{h}$. Springer, New York

Engel H. et al., 2010, ApJ, 724, 233

Feruglio C., Maiolino R., Piconcelli E., Menci N., Aussel H., Lamastra A., Fiore F., 2010, A\&A, 518, L155

Garwood R. W., Helou G., Dickey J. M., 1987, ApJ, 322, 88

George R. D. et al., 2014, MNRAS, 442, 1877

Gioia I. M., Gregorini L., Klein U., 1982, A\&A, 116, 164

Haan S. et al., 2011, AJ, 141, 100

Hayward C. C., Narayanan D., Kereš D., Jonsson P., Hopkins P. F., Cox T. J., Hernquist L., 2013, MNRAS, 428, 2529

Heckman T. M., Lehnert M. D., Strickland D. K., Armus L., 2000, ApJS, 129,493

Hekatelyne C. et al., 2018a, MNRAS, 474, 5319

Hekatelyne C. et al., 2018b, MNRAS, 479, 3966

Hoopes C. G., Walterbos R. A. M., Rand R. J., 1999, ApJ, 522, 669

Hopkins P. F., Hernquist L., Cox T. J., Di Matteo T., Robertson B., Springel V., 2006, ApJS, 163, 1

Hung C.-L., Hayward C. C., Smith H. A., Ashby M. L. N., Lanz L., MartínezGalarza J. R., Sanders D. B., Zezas A., 2016, ApJ, 816, 99

Hwang H. S., Elbaz D., Lee J. C., Jeong W.-S., Park C., Lee M. G., Lee H. M., 2010, A\&A, 522, A33

Kartaltepe J. S. et al., 2012, ApJ, 757, 23

Kauffmann G. et al., 2003, MNRAS, 346, 1055

Kewley L. J., Dopita M. A., Sutherland R. S., Heisler C. A., Trevena J., 2001, ApJ, 556, 121

Kormendy J., Ho L. C., 2013, ARA\&A, 51, 511

Lahav O., Liddle A. R, 2014, preprint (arXiv:1401.1389)

Lotz J. M., Jonsson P., Cox T. J., Primack J. R., 2008, MNRAS, 391, 1137

Lo K. Y., 2005, ARA\&A, 43, 625

Martin J.-M., Bottinelli L., Gouguenheim L., Le Squeren A.-M., Dennefeld M., 1989, C. R. Acad. Sci., Paris II, 308, 287

Martin S. J., 1989, PhD thesis, Univ. de Paris VII

Martin C. L., 2005, ApJ, 621, 227

McBride J., Heiles C., 2013, ApJ, 763, 8

Migenes V., Coziol R., Cooprider K., Klöckner H.-R., Plauchu-Frayn I., Islas J. M., Ramírez-Garduño L., 2011, MNRAS, 416, 1267

Mihos J. C., Hernquist L., 1996, ApJ, 464, 641

Monreal-Ibero A., Arribas S., Colina L., 2006, ApJ, 637, 138

Monreal-Ibero A., Arribas S., Colina L., Rodríguez-Zaurín J., AlonsoHerrero A., García-Marín M., 2010 , A\&A, 517, A28

Murtagh F., Heck A., 1987, Principal Components Analysis, in Multivariate Data Analysis. Astrophysics and Space Science Library, Vol. 131. Springer, Dordrecht, p. 236

Nardini E., Risaliti G., Watabe Y., Salvati M., Sani E., 2010, MNRAS, 405, 2505 
Osterbrock D. E., Ferland G. J., 2006, in Osterbrock D. E., Ferland G. J., eds, Astrophysics of gaseous nebulae, active galactic nuclei, 2nd edn. University Science Books, Sausalito, CA

Peng C. Y., Ho L. C., Impey C. D., Rix H.-W., 2002, AJ, 124, 266

Planck Collaboration XVI, 2013, A\&A , 571, A16

Ricci T. V., Steiner J. E., Menezes R. B., 2011, ApJ, 734, L10

Rich J. A., Torrey P., Kewley L. J., Dopita M. A., Rupke D. S. N., 2012, ApJ, 753, 5

Rich J. A., Kewley L. J., Dopita M. A., 2015, ApJ, 221, 28

Riffel R. A., 2010, Ap\&SS, 327, 239

Rossa J., Dettmar R.-J., 2000, A\&A, 359, 433

Rossa J., Dettmar R.-J., 2003, A\&A, 406, 505

Rupke D. S., Veilleux S., Sanders D. B., 2002, ApJ, 570, 588

Rupke D. S. N., Veilleux S., 2013, ApJ, 768, 75

Sales D. A. et al., 2015, ApJ, 799, 25

Sanders D. B., Mirabel I. F., 1996, ARA\&A, 34, 749

Sanders D. B., Soifer B. T., Elias J. H., Madore B. F., Matthews K., Neugebauer G., Scoville N. Z., 1988, ApJ, 325, 74

Silk J., Mamon G. A., 2012, Res. Astron. Astrophys., 12, 917

Soifer B. T. et al., 1984, ApJ, 278, L71
Spoon H. W. W. et al., 2013, ApJ, 775, 127

Springel V., Di Matteo T., Hernquist L., 2005, MNRAS, 361, 776

Steiner J. E., Menezes R. B., Ricci T. V., Oliveira A. S., 2009, MNRAS, 395,64

Sturm E. et al., 2011, ApJ, 733, L16

Tacconi L. J. et al., 2008, ApJ, 680, 246

Tarchi A., Castangia P., Henkel C., Surcis G., Menten K. M., 2011, A\&A, 525, A91

van Dokkum P. G., 2001, PASP, 113, 1420

Veilleux S., Osterbrock D. E., 1987, ApJS, 63, 295

Veilleux S., Kim D.-C., Sanders D. B., 2002, ApJS, 143, 315

Veilleux S. et al., 2009, ApJS, 182, 628

Veilleux S. et al., 2013, ApJ, 776, 27

Wagner J., 2013, A\&A, 560, A12

Wang L., Rowan-Robinson M., 2009, MNRAS, 398, 109

Wiggins B. K., Migenes V., Smidt J. M., 2016, ApJ, 816, 55

This paper has been typeset from a $\mathrm{T}_{\mathrm{E}} \mathrm{X} / \mathrm{LAT}_{\mathrm{E}} \mathrm{X}$ file prepared by the author. 